\title{
Modelling Entrainment of Sedimentary Particles by Wind and Water: A Generalized Approach
}

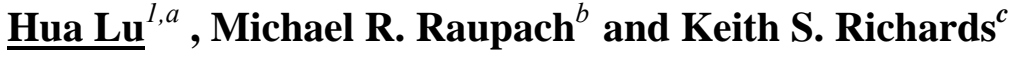 \\ ${ }^{1}$ British Antarctic Survey, Cambridge, UK \\ ${ }^{a}$ The Institute of Theoretical Physics, University of Cambridge, UK \\ ${ }^{b}$ CSIRO Marine and Atmospheric Research, Canberra, AUSTRALIA \\ ${ }^{c}$ Department of Geography, University of Cambridge, UK
}

\begin{abstract}
For longstanding theoretical reasons, it is often asserted that the threshold shear stress for entrainment of sedimentary particles $\left(\tau_{*_{t}}=\rho_{f} u_{*_{t}}^{2}\right.$, made dimensionless as $\left.A=\rho_{f} u_{*_{t}}^{2} /\left(\left(\rho_{p}-\rho_{f}\right) g d\right)\right)$, has a universal relationship with the particle Reynolds number $\left(\operatorname{Re}_{*_{t}}=u_{*_{t}} d / v\right)$, where $u_{*_{t}}$ is the threshold friction velocity, $\rho_{f}$ is the fluid density, $\rho_{p}$ is the density of the particles, $d$ is the particle diameter, $g$ is the gravitational acceleration and $v$ is the kinematic viscosity of the fluid. However, experimental plots of $A\left(\mathrm{Re}_{*}\right)$ for sediment entrainment in air and water show two major differences: (1) for large $\mathrm{Re}_{*}$, the values of $A$ in water are, in general, a few times larger than those in air, and (2) when $\operatorname{Re} *_{t}<1, A$ increases more rapidly in air than in water as $\mathrm{Re}_{*}{ }_{t}$ decreases. This paper derives a new, general theory for $A$, which incorporates the effects of fluid turbulence, particle cohesion and probabilistic aspects of grain entrainment. It is found that difference (1) is explained by differences in the probability distribution of streamwise velocity fluctuations for typical situations in air and water, which follow from basic scaling laws for velocity variances in turbulent flow. Difference (2) is explained by the different behaviours of interparticle cohesion forces in air and water. The resulting expression is shown to compare well with experimental data.
\end{abstract}

Keywords: sediment; entrainment; threshold shear stress; threshold velocity; near-surface turbulent fluctuations. 


\section{Introduction}

The prediction of incipient motion of a particle lying in a bed of similar particles (i.e. the entrainment of sedimentary particles from the surface into the fluid when the fluid speed exceeds a certain threshold), is fundamental for understanding sediment transport by water and wind [Graf, 1971; Quan and Wan, 1983; Nickling, 1988; Raupach and Lu, 2004]. Shields [1936] pioneered research into incipient motion in open channels by introducing the dimensionless threshold shear stress $A=\tau_{*_{t}} /\left(\rho_{p}-\rho_{f}\right) g d$, a measure of the ratio of the threshold hydrodynamic force on a surface particle to its weight (see Table 1 for definitions of symbols). Shields argued that $A$ should be a unique function of the particle Reynolds number $\operatorname{Re}_{*_{t}}=u *_{t} d / v$, as

$$
A=F\left(\operatorname{Re}_{*_{t}}\right) .
$$

For particle entrainment by air flow, Bagnold [1941] derived a similar theory considering the balance between the aerodynamic drag and the gravitational force. He found that at large $\operatorname{Re}_{*}, A$ is nearly a constant and $u *_{t} \propto d^{1 / 2}$, which is essentially consistent with Shields' theory obtained in open channels. Across all particle sizes, Bagnold also assumed that $A$ is a unique function of $\operatorname{Re}_{*}$, again consistent with the proposals of Shields.

Over the last 70 years, experimental research into incipient motion has in general supported the theories of Shields and Bagnold [White, 1970; Graf, 1971; Yalin, 1972; Mantz, 1977; Buffington and Montgomery, 1997; Zingg, 1953; Greeley and Iversen, 1985; Nickling, 1988]. Figure 1 shows data obtained in experiments in air [Cleaver and Yates, 1973; Fletcher, 1976a,b; Iversen and White, 1982; ] and in water [Graf, 1971; Yalin, 1972; White, 1970], plotted on a typical Shields' $A \sim \operatorname{Re}_{*}$ diagram. Three distinct regions can be identified:

i) $\quad \operatorname{Re}_{*_{t}} \geq 10$; a region where the flow is fully turbulent, and where $A$ attains a constant value of approximately 0.04 to 0.06 at $\operatorname{Re}_{*} \geq 500$ in water, and 0.01 to 0.03 in air (note that there are few data with $\operatorname{Re}_{*} \geq 200$ for air flows);

ii) $\quad \operatorname{Re}_{*_{t}}<1$; a region where particle entrainment is mainly due to viscous laminar flow, where $A$ increases while $\mathrm{Re}_{* t}$ decreases, at a rate which is steeper in air than in water; and

iii) A transitional region, $1 \leq \operatorname{Re}_{*_{t}} \leq 10$, where the laminar sublayer partially covers the particles, but the outer turbulent flow is partially affected by the roughness of the grain bed. Within this region, $A$ reaches a minimum value of approximately 0.03 to 0.06 for water [Yalin and Karahan, 1979], but such minima are not obvious for air entrainment [Greeley and Iversen, 1985]. 
Figure 1 shows that data obtained from airflow and open channel flow do not collapse to a single $A$ $\sim \operatorname{Re}^{*}{ }_{t}$ relationship. The upturn of the Shields curve at $R e^{*}{ }_{t}<1$ in air flow is much sharper than for water flow. For $R e_{*} \geq 5, A$ is about 3 to 4 times smaller in air than in water.

\section{[[Insert Figure 1 here]]}

Several researchers have noted the different behaviour of $A$ in air and in water, and that the expressions for $A$ derived in one fluid cannot be directly applied to another [Bagnold, 1941; Graf, 1971; Iversen and Pollack, 1987]. Bagnold [1941] suggested that the differences might be due to a difference in surface texture or bed packing conditions, or to observation errors. Iversen and Pollack [1987] attributed the difference to density ratio differences. By adding the impacting force of saltating particles to the force balance equation, they related $A$ empirically to the particle-to-fluid density ratio. Although not directly targeting the different behaviour of $A$ in air and in water, a recent study of incipient motion in air has also incorporated the effect of the particle-to-fluid density ratio [Cornelis and Gabriels, 2004]. Interestingly, although Cornelis and Gabriels [2004] and Iversen and Pollack [1987] both related $A$ to the density ratio, and both utilized the same data of Iversen and White [1982], the expression of Iversen and Pollack [1987] suggests that $A$ decreases with increasing particle-to-fluid density ratio while the expression of Cornelis and Gabriels [2004] suggests $A$ increases with increasing density ratio. Making the situation more confusing, a comprehensive review of incipient motion in open channel flow concludes that there is no obvious dependence of $A$ on particle density, therefore, on density ratio [Buffington and Montgomery 1997]. These contradictions suggest that the quantitative relationships between $A$ and flow conditions, fluid properties and particle properties remain unclear. It is also not clear why, for a given fluid and under similar experimental conditions, randomly-varying values of $A$ have often been obtained. Much research into incipient motion has been based on a force balance at the instant of particle entrainment [Bagnold, 1941; Phillips, 1980; Iversen and White, 1982], and/or analysis of dimensionless groups [Shields, 1936; Fletcher, 1976a,b]. With certain assumptions, the functional forms for the parameter $A$ are predetermined and then fitted to experimental data, and in some cases, excessive parameter fitting is involved [Greeley and Iversen, 1985; Cornelis and Gabriels, 2004]. These studies have provided a means of estimating threshold shear stress under idealized situations. Nevertheless, such a deterministic reductionism often results in partial explanations of the several processes involved in incipient motion. Apart from the inability to explain differences of $A \sim \operatorname{Re}_{* t}$ relationships in air and water, there is a lack of agreement about the values of $A$ and the physical causes of why, for $\mathrm{Re}_{*} \leq 1$, the estimates of $A$ vary by an order of magnitude or more for a given $\mathrm{Re}_{*}$. Emphasizing the effects of flow, Bagnold [1956] argued that $A$ will not exceed 0.4, but values 
of $A$ greater than 10 have been found for $d \leq 10 \mu \mathrm{m}$ [White, 1970; Cleaver and Yates, 1973; Fletcher, 1976a,b]. By assuming laminar sub-layer flow, Yalin [1972] derived $A=0.1 / \operatorname{Re}_{*}$. With similar assumptions for the flow regime and force balance, Ling [1995] suggested that $A$ is inversely proportional to $\mathrm{Re}_{*_{t}}$ for rolling grains, and inversely proportional to $\mathrm{Re}_{*_{t}}^{2}$ for a threshold lift condition. Both studies implied that the increase in $A$ with decreasing $\operatorname{Re} *_{t}$ is solely due to the transition in flow regime from turbulent to laminar flow.

For particle entrainment in air flow, Iversen and White [1982] attributed the sharp upturn of $A$ for $\operatorname{Re}_{*_{t}}<1$ to the effects of inter-particle cohesion forces. To account for the effect of cohesion, Greeley and Iversen [1985] suggested that $A$ should be of the form

$$
A=A_{1} F\left(\mathrm{Re}_{*_{t}}\right) G(d)
$$

where the dimensionless functions $F\left(\mathrm{Re}_{*}\right)$ and $G(d)$ respectively represent the effects of aerodynamic and cohesion forces. Eq. (2) essentially implies that the upturn of $A$ for $\operatorname{Re}_{*}<1$ is partially due to the flow condition (through the term $F\left(\operatorname{Re}_{*}\right)$ ) and partially due to inter-particle cohesion (through the term $G(d)$ ). Though eq. (2) overcomes the shortcomings of the early expression of Bagnold [1941] and is effective in describing the behaviour of $u_{*}$ for the entire particle size range, the expressions proposed by Greeley and Iversen [1985] involve the two empirical functions, $F\left(\operatorname{Re}_{t}\right)$ and $G(d)$, and are difficult to relate to physical processes.

While earlier theoretical analysis often focused on the effects of flow conditions by relating $A$ to the particle Reynolds number $\mathrm{Re}_{*}$, more recent empirical or semi-empirical studies have to some degree sought to remove the flow term $F\left(\operatorname{Re}_{*}\right)$ in eq. (2), or to replace it by a particle property. For instance, Marticorena and Bergametti [1995] simplified the expressions of Greeley and Iversen [1985] by expressing $\mathrm{Re}_{*}$ as a function of particle size only. Shao and $L u$ [2000] reanalyzed the wind tunnel data of Iversen and White [1982] and suggested that $A=A_{N} G(d)$, where $A_{N}$ is a constant of 0.013 and $G(d)$ representing the effects of interparticle cohesion,. More recently, Cornelis and Gabriels [2004] expressed $A$ as a function of the particle-to-fluid density ratio, particle diameter and interparticle cohesion. Their expression involves five empirical constants that were determined by fitting to the data of Iversen and White [1982] using non-linear regression. Such emphases on sedimentological controls are also abundant in the hydraulic literature. For example, in open channels with mixed bed material particle sizes, studies have commonly expressed $A$ as an inverse empirical function of the ratio of particle size $d$ to the underlying median bed particle size $d_{50}$ [Komar and Li, 1988; Richards, 1990; Buffington and Montgomery, 1997]. 
A clear advantage of using these semi-empirical expressions of $A$ is their simplicity. If $A$ is not expressed as a function of $\operatorname{Re}_{*}$, iteration is avoided, since the friction velocity $u *_{t}$ no longer appears on both sides of the expression for $u_{*}$. This is particularly appealing for large-scale spatiallydistributed applications [Marticorena and Bergametti, 1995]. However, the drawback of these expressions is their failure to account fully for the physical processes involved. As noted by Qian and Wan [1983], a variety of models could fit the experimental data well as long as the chosen expression has the right shape and contains at least two free parameters to describe the upturn of $A$ for both large and small $\mathrm{Re}_{*}{ }_{t}$ ranges. There is little basis for judging the superiority of an expression solely on goodness of statistical fit if the physical meaning of the fitted parameters is not clearly understood. A more general, physically-based approach to the threshold of entrainment is needed. Realizing that deterministic approaches can only estimate the average values of threshold shear stress but unable to predict the observed variations, some researchers have proposed that particle incipient motion is a stochastic process, i.e. there exists a range of threshold shear stresses for a given particle size [Grass, 1970; Gessler, 1971]. Field and laboratory observations in open channel flow confirm a variability of threshold shear stress attributable to a number of random factors including temporal fluctuation of near bed turbulence, the bed packing condition, and heterogeneities in grain size and shape, etc [Einstein, 1950; Grass, 1970]. In the hydraulic literature, recent research into incipient motion has focused on finding appropriate probability distributions for instantaneous flow velocity or shear stress [Cheng and Chiew, 1998; Wu and Lin, 2002], the effects of random bed roughness and picking conditions [Papanicolaou et al., 2001; 2002], or the combined probabilistic nature of turbulent fluctuation and bed grain geometry [ $\mathrm{Wu}$ and $\mathrm{Chou}, 2003]$. Nevertheless, incorporating statistical/stochastic concepts remains rare in the modeling of particle incipient motion during wind erosion.

The objective of this paper is to derive a general expression for $A$, to explain the differences in $A \sim$ $\mathrm{Re}_{*}{ }_{t}$ plots between air and water. We incorporate the effect of fluid turbulence by utilizing recent advances in understanding of turbulence over rough walls, especially the scaling of velocity fluctuations with bulk Reynolds number (using boundary layer height $\delta$ as the length scale rather than particle diameter $d$ ). We also consider the probabilistic aspects of grain entrainment by adopting a log-normal distribution for the near-bed instantaneous flow velocity. The new expression also incorporates the influences of interparticle cohesion forces to account for the upturning of $A$ for small values of $\operatorname{Re}_{*}$. We use the new expression to analyze the effects of both the mean flow and turbulent fluctuations, and the physical causes of the different regions in the Shields 
diagram, which are shown to depend on changes in the relative magnitude of the appropriate forces. Though no direct curve-fitting is used, our results compare well with available experimental data.

\section{Methods and The New Theory}

This paper considers a flat surface covered by uniformly-sized erodible particles. The $x$-axis lies in the plane of the bed surface, its direction parallel to the direction of flow, while the $y$-axis is perpendicular to the bed and directed upwards. The height origin $(y=0)$ is taken to be the aerodynamic height origin, defined as the level of effective drag or zero-plane displacement (Figure 2). An overbar denotes a temporal average. A superscript ${ }^{+}{ }^{+}$with a velocity variable (either its mean or fluctuation) denotes normalization by the friction velocity $u *$, as in $u^{+}=u / u_{*}$; a similar superscript with a length variable denotes normalization by the viscous length scale $v / u *$, as in $y^{+}=u_{*} y / v$. Also, subscripts $a$ and $w$ denote the air and water cases, respectively.

\section{[[Insert Figure 2 here]]}

\subsection{Forces Involved in Particle Entrainment}

Analysis of the particle threshold condition begins by defining a force balance on a static grain sitting on the bed surface. A particle resting on the bed (Figure 2) is subjected to forces of $\operatorname{drag} F_{D}$, lift $F_{L}$, specific weight $F_{G}$ and a net cohesion force $F_{C}$. We assume that $F_{C}$ is approximately equal in magnitude to the cohesion force between two adjacent individual particles, but follows the direction of $F_{G}$. The forces other than $F_{C}$ can be expressed as

$$
\begin{aligned}
& F_{G}=\frac{\pi}{6}\left(\rho_{p}-\rho_{f}\right) g d^{3}, \\
& F_{D}=C_{D} \frac{\rho_{f} S u_{\Delta}^{2}}{2} \\
& F_{L}=C_{L} \frac{\rho_{f} S u_{\Delta}^{2}}{2}
\end{aligned}
$$

where $u_{\Delta}=\bar{u}+u$ ' is a streamwise reference instantaneous velocity at the threshold of grain motion, and other symbols are defined in Table 1 . The frontal area $S$ is approximately equal to $0.2 \pi d^{2}$ for the packing geometry shown in Figure 2. 
Choosing the reference height $y_{\Delta}$ where $u_{\Delta}$ is defined can be difficult. Wu and Lin [2002] and Wu and Chou [2003] effectively calculated $y_{\Delta}$ by integrating both $\bar{u}$ and $u^{\prime}$ over the frontal area of the about-to-move particle exposed to the flow. Such an approach is difficult to justify in fluid mechanical terms, for several reasons. Firstly, one cannot define the instantaneous velocity at a solid surface as zero, because of the no slip condition. Secondly, at any height within the roughness elements, the velocity field is complex and three-dimensional because fluid has to find its way around the roughness elements. Thirdly, the drag on a surface-mounted roughness element does not satisfy a simple momentum integral constraint equivalent to the relationship for a wake of an obstacle in a free stream [Batchelor, 1967; Raupach, 1992], because of the absence of a free-stream velocity. Finally, an individual roughness element is exposed to a turbulent flow including not only a mean shear but also strong turbulence with contributions both from the large-scale boundary-layer and also the wakes of other roughness elements.

To handle these complexities, it is usual to define $u_{\Delta}$ as the velocity at a reference height $y_{\Delta}$, horizontally-averaged over an area large enough to smooth out spatial fluctuations caused by the bed roughness (in practice a horizontal distance of order 10d). This quantity is analogous to the free stream velocity for the drag coefficient of a body in a uniform flow. In the case of incipient motion, the logical choice for $y_{\Delta}$ is the mean height of the most exposed roughness elements, i.e. $y_{\Delta}=\beta d$, where $\beta$ is a constant. For instantaneous velocity, the likely choice for $\beta$ is between 0.5 and 1.0, since the most exposed elements rest some way above the mean position of all bed elements. The level of effective drag or zero-plane displacement (our height origin $\mathrm{y}=0$ ) is approximately $0.5 d$ to 0.7d above the bed substrate level [Jackson, 1981; Bridge and Bennett, 1992]. Cheng and Chiew [1998] suggested $\beta=0.6$ for the most stable bed packing situation where particles of identical size rest in an interstice formed by closely-packed bed surface particles. In Section 3.1, the effects of $\beta$ on the values of $A$ will be discussed.

The effects of cohesion forces on incipient motion and the dependence of cohesion forces on particle size are complex and poorly understood [Zimon, 1982; Shao and Lu, 2000; Cornelis and Gabriels, 2004]. Cohesion forces arise from both mutual attraction between solid particles, and interaction between the solid particles and those of the ambient fluid medium. The cohesion force is affected by the combination of molecular forces (including the van der Vaal's forces), Coulomb forces, electrostatic forces, capillary forces, and chemical bonding forces. Although these forces are functions of particle size $d$, their dependences on $d$ are different, with molecular forces and electrostatic forces proportional to $d$, Coulomb forces proportional to $1 / d^{2}$, the capillary force 
proportional to $d\left(1-d^{x-1}\right)$, where $x$ is the width of a microscopic water bridge in the contact zone of two particles [Zimon, 1982]. For instance, the cohesion forces between two identical spheres can be expressed as

$$
F_{C}=\frac{c_{i}}{32 \pi z_{i}^{2}} d
$$

where $c_{i}$ is the cohesion coefficient and $z_{i}$ is the smallest separation between two spherical particles [Theodoor and Overbeek, 1985]. Eq. (6) indicates two possible relationships between $F_{C}$ and $d$ : if $z_{i}$ is proportional to $d$, Eq (6) gives $F_{C}$ proportional to $1 / d$ (assuming no dependence of $c_{i}$ on $d$ ), while if $z_{i}$ is a constant, $F_{C}$ is proportional to $d$.

Observations in air have resulted in various relationships between $F_{C}$ and $d$ : direct, inverse and exponential dependence of the cohesion force on $d$, or even complete independence over a certain range of $d$ [Zimon, 1982]. In water, the dependence of cohesion forces on particle size is simpler and most experiments suggest that $F_{C}$ is proportional to $d$ [Corn, 1961; Zimon, 1982]. This is because in water, capillary and electrostatic forces do not apply as any charges on the particles will leak away and no capillary bridge develops within the particle contact zone [Theodoor and Overbeek, 1985]. This results in the cohesion force in a liquid medium being governed by molecular forces, which implies direct proportionality to $d$ [Fuks, 1955]. Nevertheless, in the case of threshold conditions over a loosely-packed bed with a small removal probability of grains (approximately $2 \%$ ), direct proportionality between $F_{C}$ and $d$ may hold in both water and air media as

$$
F_{C}=C_{C} d,
$$

where $C_{C}$ is a proportionality constant, according to Fuks [1955] and Zimon [1982].

At the instant of particle motion, the combined retarding force moments must just balance the combined hydrodynamic driving force moment. Such a condition is expressed as

$$
F_{D} L_{D}+F_{L} L_{L}=F_{G} L_{G}+F_{C} L_{C}
$$

where $L_{D}=d \cos \theta, L_{L}=d \sin \theta, L_{G}=d \sin \theta$ and $L_{C}=d \sin \theta$ are the moment arms (about the pivot point P) of $F_{D}, F_{L}, F_{G}$ and $F_{C}$ (as shown in Figure 2). Following $W u$ and Lin [2003], the pivoting angle $\theta$ ranges from $30^{\circ}$ when the grain is at its most exposed, to $90^{\circ}$ when it is embedded within the surface grain layer.

Combining eqs. (3) to (5) and (7) with eq. (8), the threshold condition can be written as 


$$
u_{\Delta} \geq u_{t}
$$

where

$$
u_{t}=\sqrt{\frac{2 L_{w}}{C_{D} L_{D}+C_{L} L_{L}} \frac{\pi d^{3}}{6 S} \frac{\left(\rho_{p}-\rho_{f}\right) g}{\rho_{f}}\left(1+\frac{\rho_{f}}{\left(\rho_{p}-\rho_{f}\right) g} \frac{6 C_{C} L_{C}}{\pi L_{w}} \frac{1}{d^{2}}\right)}
$$

is the threshold velocity. Eq. (9) simply states that the instantaneous reference velocity $u_{\Delta}$ must exceed $u_{t}$ for particle detachment to occur. If we assume $u_{\Delta}$ obeys a certain probability distribution, $u_{t}$ is therefore defined by a point on the velocity probability distribution of $u_{\Delta}$ at the reference height $y$ from the bed where $u_{\Delta}$ is defined, and the probability of particle detachment from the bed is $p=\operatorname{Prob}\left(u_{\Delta} \geq u_{t}\right)$, as shown in Figure 3. The derivation of $A$ based on a log-normal distribution of $u_{\Delta}$ for a given probability removal rate $p$ is given in Section 2.4.

\section{[[Insert Figure 3 here]]}

We turn now to the values of $C_{D}$ and $C_{L}$. Compared with the well-studied situation of an isolated object in a free stream, where the drag coefficient is a function of Reynolds number $\left(\operatorname{Re}_{*}=u * d / v\right)$ and is proportional to $1 / \mathrm{Re}_{*}$ for small $\mathrm{Re}_{*}$, the drag coefficient for a particle resting on a surface is more complex and less well understood [Fischer et al., 2002; Jiménez, 2004]. It has been found that the drag coefficient $C_{D} \approx 0.15-0.30$ for a sphere resting on a surface [Tillman, 1944], but may be up to 1.25 for two-dimensional span-wise obstacles [Jiménez, 2004]. Chepil [1958] provided one of the most comprehensive data sets on the lift coefficient $C_{L}$ in air, and showed that the average ratio of lift to drag is nearly constant at 0.85 for a boundary layer friction Reynolds number of $\operatorname{Re}_{\tau} \leq$ 5000, where $\operatorname{Re}_{\tau}=u * \delta / v$ and $\delta$ is the height of the boundary layer. Mainly for channel flows, James [1990] proposed

$$
\frac{C_{L}}{C_{D}}=\left\{\begin{array}{ll}
-0.56+0.212 \ln \mathrm{Re}_{*} & \mathrm{Re}_{*}<150 \\
0.5 & \mathrm{Re}_{*} \geq 150
\end{array} .\right.
$$

Eq. (11) suggests that $C_{L}$ could be negative at $\mathrm{Re}_{*}<15$. Marsh et al. [2004] derived a similar expression to eq. (11) but with $C_{L} / C_{D} \approx 0.2$ for $\mathrm{Re}_{*}>100$. The data of Patnaik et al. [1994] for gravel-bed rivers showed $C_{L} / C_{D} \approx 1$, and an apparent decreasing trend of the lift-to-drag ratio with increasing $\mathrm{Re}_{*}$ in the range 4,000 to 60,000. However, this has limited relevance to this study, where we focus on $\mathrm{Re}_{*}$ in the range from 1 to 10,000 . This is because aeolian transport involves a narrower range of particle sizes than in rivers, and in air, there is little reliable data on threshold velocities for $\mathrm{Re}_{*_{t}}>100$. Furthermore, the exact values for temporally-averaged drag and lift 
coefficients are unknown at present, as they both depend upon flow conditions. Though the dependence of $C_{D}$ and $C_{L}$ on $\mathrm{Re}_{*}$ is likely to have some effects on $A$, especially for small $\mathrm{Re} *$, for simplicity, values of $C_{D}=0.5$ and $C_{L}=0.3$ are used in this study. According to Coleman [1967], for a $3 \mathrm{D}$ sphere and $\theta=30^{\circ}, L_{D}=d \sqrt{3} / 2$, and $L_{L}=L_{G}=L_{C}=d / 2$. These values are used here.

\subsection{Mean Velocity Profile over Rough Surfaces}

The classic theory of near-wall turbulent flow defines the universal logarithmic velocity profile for a smooth wall,

$$
\bar{u}^{+}=\frac{1}{\kappa} \ln y^{+}+B
$$

where $\bar{u}$ is the mean flow velocity at height $y, \kappa$ is von Karman's constant (0.41), and $B$ is an empirical constant with a value of 5.0 [Clauser, 1956; Panton, 2005]. The effect of roughness on the logarithmic velocity profile is a downward shift of eq. (12), corresponding to the increase in skin friction [Raupach et al., 1991]. The logarithmic law for a rough surface can then be written as

$$
\bar{u}^{+}=\frac{1}{\kappa} \ln y^{+}+B-\Delta \bar{u}^{+} .
$$

There are three regimes in turbulent flow: smooth, transitionally-rough and fully-rough flow [Raupach et al., 1991]. Ligrani and Moffat [1986] suggested that these three flow regimes can be classified by $k_{s}^{+}<2.25$ when the flow is smooth, and $k_{s}^{+}>90$ when it is fully rough, where $k_{s}^{+}=u_{*} k_{s} / v$ is the roughness Reynolds number, and $k_{s}$ is the equivalent sand grain roughness.

The roughness function $\Delta \bar{u}^{+}$in eq. (13) has been measured experimentally by Nikuradse [1933] using sand roughness of different grain sizes, and in many other experiments over other kinds of rough surface; see Raupach et al. [1991] for review. For a uniform sand bed, $k_{s}$ is equal to the diameter of the particles. Ligrani and Moffat [1986] used Nikuradse's data to obtain

$$
\Delta \bar{u}^{+}=\xi\left(\frac{1}{\kappa} \ln k_{s}^{+}+B-8.5\right),
$$

in which the interpolation function

$$
\xi= \begin{cases}0 & k_{s}^{+}<2.25 \\ \sin \left[\frac{\pi \ln \left(k_{s}^{+} / 2.25\right)}{2 \ln (90 / 2.25)}\right] & 2.25 \leq k_{s}^{+}<90 \\ 1 & k_{s}^{+} \geq 90\end{cases}
$$


increases from 0 to 1 through the transitionally-rough regime, $2.25 \leq k_{s}^{+}<90$. Eq. (14) recovers the smooth wall log-law in the smooth regime, and suggests that, in the transitionally-rough regime, the roughness function $\Delta \bar{u}^{+}$is near zero for $k_{s}^{+}$below approximately 5 .

We note in passing that the rough wall law, eq. (13), is commonly represented as

$$
\bar{u}^{+}=\frac{1}{\kappa} \ln \left(\frac{y}{z_{0}}\right),
$$

where $z_{0}$ is the zero-velocity level of the logarithmic profile. The dimensionless quantities $z_{0} / k_{s}, \xi$ and $\Delta \bar{u}^{+}$carry equivalent information [Raupach et al., 1991]. Substituting eqs. (13) and (14) into eq. (16) and solving for $z_{0}$, we have

$$
\begin{aligned}
z_{0} & =k_{s} \exp \left[-\ln k^{+}-\kappa\left(B-\Delta \bar{u}^{+}\right)\right] \\
& =k_{s} \exp \left[(\xi-1) \ln k_{s}^{+}+(\xi-1) \kappa B-8.5 \kappa \xi\right]
\end{aligned}
$$

For fully rough flows, $\xi=1$ (eq. (15)). Substituting it into eqs. (13) and (17) gives

$$
\bar{u}^{+}=\frac{1}{\kappa} \ln \left(\frac{y}{k_{s}}\right)+8.5,
$$

and

$$
z_{0} / k_{s}=\exp (-8.5 \kappa)=0.031
$$

respectively. Eqs. (18) and (19) are consistent with common assumptions in the hydraulics literature and the ' $z_{0} \sim d / 30$ rule' that is commonly known to wind tunnel experimentalists (assuming $d=k_{\mathrm{s}}$ for well sorted sand). In terms of threshold entrainment, all three flow regimes are relevant, depending on particle size and flow conditions. In this study, we shall compare the dimensionless shear stress $A$ resulting from eqs. (13) and (14) with those derived from the fully-rough limit, eqs. (16) and (19).

Some researchers argue that $\bar{u}^{+}$depends not only on $y^{+}$but also on the flow Reynolds number [Barenblatt and Chorin, 1998]. However, in comparison to that of roughness, such effects are relatively minor in the inner part of the mean velocity profile [Panton, 2005] and are not fully understood when roughness elements are present [Bergstrom et al., 2001]. 


\subsection{Reynolds-Number Dependence of Near-bed Turbulent Velocity}

Like the mean velocity profile, the turbulence in air and in water is governed by universal dimensionless scaling laws. However, a major distinction between turbulent airflow and turbulent river flow lies in the flow Reynolds number $\left(\operatorname{Re}_{\tau}=u_{*} \delta / \nu\right.$, where $\delta$ is the boundary layer depth). Under the conditions of incipient motion, the difference is mainly due to the intrinsic length scale $\delta$, as the difference in viscous length scale $u * v$ of the two media is relatively small. At the threshold condition, the boundary layer depth in a typical wind tunnel is of the order of 1 metre or more [Greeley and Iversen, 1985], whereas it is of the order of a few centimetres in laboratory flumes [Graf, 1971; Yalin, 1972]. The Reynolds number in the atmospheric boundary layer is about 1001000 times higher than that observed in natural rivers, whereas the sub-layer thickness remains nearly identical [Metzger and Klewicki, 2001]. We shall show that these differences are one of the reasons for the systematically smaller values of the dimensionless shear stress $A$ observed in air relative to water (Figure 1).

\section{[[Insert Figure 4 here]]}

The turbulent velocity (here considered to be the standard deviation of the streamwise velocity, $\sigma=\sqrt{\overline{u^{\prime 2}}}$ ) is a strong function of the Reynolds number [DeGraaff and Eaton, 2000; Metzger and Klewicki, 2001; Marusic and Kunkel, 2003]. As shown in Figure 4, there is a clear dependence of $\sigma^{+}=\sigma / u_{*}$ on the flow Reynolds number $\operatorname{Re}_{\tau}$ across all values of the inner variable $y^{+}$. Marusic and Kunkel [2003] proposed a scaling formulation to account for the full range of turbulence intensity $\sigma^{+}$in relation to $y^{+}$and $\operatorname{Re}_{\tau}$, based upon the attached eddy hypothesis and the idea that the attached eddy motions in the log region and beyond impose a forcing on the viscous buffer zone and sublayer:

$$
\left(\sigma^{+}\right)^{2}=\frac{\sigma^{2}}{u_{*}^{2}}= \begin{cases}\frac{0.16\left(y^{+}\right)^{2}}{\left(1+b_{1}\left(y^{+}\right)^{2}\right)^{1 / 2}\left(1+\left(b_{2} y^{+}\right)^{2 b_{3}}\right)^{1 / 2}}\left[1+(\alpha-1) \frac{\ln y^{+}}{\ln 50}\right] & y^{+} \leq 30 \\ B_{1}-B_{2} \ln \left(\frac{y^{+}}{\operatorname{Re}_{\tau}}\right)-B_{3}\left[1-\left(y^{+}\right)^{-0.9}\right]\left(y^{+}\right)^{-0.5} & y^{+} \geq 150\end{cases}
$$

where $\alpha=\frac{B_{1}-B_{2} \ln \left(50 / \mathrm{Re}_{\tau}\right)}{B_{1}-B_{2} \ln (50 / 2000)}, b_{1}=0.008, b_{2}=0.115, b_{3}=1.6, B_{1}=2.39, B_{2}=1.03$, and $B_{3}=$ 5.58. For $30 \leq \mathrm{y}^{+} \leq 150$, interpolation is needed. For simplicity, linear interpolation is used in this study. In essence, Eq. (20) describes the behaviour of $\sigma^{+}$shown in Figure 4, i.e. $\sigma^{+}$increases with 
$\operatorname{Re}_{\tau}$ and peaks at $y^{+} \approx 15$ for a given value of $\operatorname{Re}_{\tau}$. This maximum value of $\sigma^{+}$can also be expressed as a function of $\operatorname{Re}_{\tau}$

$$
\sigma_{\max }^{+}=\sqrt{0.964+0.87 \ln \left(\mathrm{Re}_{\tau}\right)}
$$

The $\sigma_{\max }^{+} \sim \operatorname{Re}_{\tau}$ relationship described by eq. (21) is plotted in Figure 5. Typical value ranges of $\operatorname{Re}_{\tau}$ for flumes, natural rivers, wind tunnels and atmospheric boundary layers are also shown. This shows that $\sigma_{\max }^{+} \approx 2.5$ for open channel flow (assuming $d=1 \mathrm{~mm}, \delta=0.015 \mathrm{~m}$ and $u_{t}=0.03 \mathrm{~m} \mathrm{~s}^{-1}$ ), and $\sigma_{\max }^{+} \approx 3.5$ for air flow in a wind tunnel (assuming $d=1 \mathrm{~mm}, \delta=1.2 \mathrm{~m}$ and $u_{*_{t}}=0.5 \mathrm{~m} \mathrm{~s}^{-1}$ ). The former value of $\sigma_{\max }^{+}$is consistent with those commonly used in the hydraulic literature [Cheng and Chiew, 1998; Wu and Lin, 2002].

\section{[[Insert Figure 5 here]]}

Though eqs. (20) and (21) were developed for smooth surfaces, recent studies show that roughness enhances the turbulence and the Reynolds shear stress over most of the boundary layer, and promotes isotropy as a result of mixing caused by the wakes generated by the roughness elements [Raupach et al. 1996; Krogstad and Antonia, 1999; Tachie et al., 2004]. However, for a sand surface, the increment of turbulence intensity is quite small for the streamwise component [Tachie at al., 2004], in contrast with surfaces of higher roughness such as vegetation. Therefore, eqs. (20) and (21) are used in our analysis.

\subsection{Entrainment Probability in a Log-Normal Distribution of Instantaneous Velocities}

The near-bed turbulence is intense and dominated by gust-like eddy motions with length scales determined by the characteristic length scale of the roughness, $k_{s}$ or $d$ [Raupach et al. 1991, 1996]. These gusts cause the streamwise velocity to show significant departure from a normal velocity distribution [Morrison et al., 2004], with strong positive skewness near the bed. It is reasonable to describe the highly positively skewed distribution for the instantaneous velocity $u_{\Delta}$ as log-normal [Wu and Lin, 2002] (Figure 3). Thus, we assume that if $v_{\Delta}$ denotes the logarithm of $u_{\Delta}$ (i.e. $\left.v_{\Delta}=\ln u_{\Delta}\right)$, the probability density function $f\left(v_{\Delta}\right)$ obeys a normal distribution:

$$
f_{v}\left(v_{\Delta}\right)=\frac{1}{\sqrt{2 \pi \sigma_{v}}} \exp \left[-\frac{\left(v_{\Delta}-\overline{v_{\Delta}}\right)^{2}}{2 \sigma_{v}^{2}}\right]
$$

where $\overline{v_{\Delta}}$ and $\sigma_{v}$ are the mean and standard deviation of $v_{\Delta}$, respectively. 
The probability of entrainment $(p)$ can be expressed as

$$
p=\operatorname{Prob}\left(u_{\Delta} \geq u_{t}\right)=\operatorname{Prob}\left(v_{\Delta} \geq v_{t}\right)=1-\operatorname{Prob}\left(-\infty<v_{\Delta}<v_{t}\right)
$$

where $v_{t}=\ln u_{t}$ and $u_{t}$ is the threshold velocity determined by eq. (10). Physically, $p$ can be interpreted as the fraction of the time over which the instantaneous reference velocity $u_{\Delta}$ must exceed the threshold velocity $u_{t}$ for detachment to occur. As will be shown later, $p$ emerges as a critical parameter in estimating $A$. Using an approximation for the error function [Cheng and Chiew, 1998], we have

$$
\begin{array}{r}
p=1-\left[\int_{-\infty}^{\overline{v_{\Delta}}} f_{v}\left(v_{\Delta}\right) \mathrm{d} v_{\Delta}+\int_{\overline{v_{\Delta}}}^{v_{t}} f_{v}\left(v_{\Delta}\right) \mathrm{d} v_{\Delta}\right] \\
0.5\left(1-\frac{v_{t}-\overline{v_{\Delta}}}{\left|v_{t}-\overline{v_{\Delta}}\right|} \sqrt{\left.1-\left[-\frac{2\left(v_{t}-\overline{v_{\Delta}}\right)^{2}}{\pi \sigma_{v}^{2}}\right]\right),}\right.
\end{array}
$$

so that

$$
v_{t}=\overline{v_{\Delta}}+\sigma_{v} \frac{0.5-p}{|0.5-p|} \sqrt{\frac{\pi}{2} \ln \left[\frac{1}{4 p(1-p)}\right]},
$$

where $0<p<1$. The mean $\overline{v_{\Delta}}$ and variance $\sigma_{v}^{2}$ of $v_{\Delta}$ can be estimated by first-order approximation using a Taylor series expansion, and then related to the mean and variance of $u_{\Delta}$, giving

$$
\begin{aligned}
& \overline{v_{\Delta}}=\ln \left[\overline{u_{\Delta}} / \sqrt{1+\left(\sigma / \overline{u_{\Delta}}\right)^{2}}\right]=\ln \left(\bar{u}_{*}{\overline{u_{\Delta}}}^{+} / \sqrt{1+\left(\sigma^{+} /{\overline{u_{\Delta}}}^{+}\right)^{2}}\right), \\
& \sigma_{v}^{2}=\ln \left[1+\left(\sigma / \overline{u_{\Delta}}\right)^{2}\right]=\ln \left[1+\left(\sigma^{+} /{\overline{u_{\Delta}}}^{+}\right)^{2}\right] .
\end{aligned}
$$

Combining eqs. (24), (26) and (27), we have

$$
u_{t}=\frac{u_{*}{\overline{u_{\Delta}}}^{+}}{\sqrt{1+\left(\sigma^{+} /{\overline{u_{\Delta}}}^{+}\right)^{2}}} \exp \left\{\frac{0.5-p}{|0.5-p|} \sqrt{\ln \left[1+\left(\sigma^{+} /{\overline{u_{\Delta}}}^{+}\right)^{2}\right]} \sqrt{\frac{\pi}{2} \ln \left[\frac{1}{4 p(1-p)}\right]}\right\} .
$$

Therefore, substituting eq. (10) into eq. (28) and solving for the threshold friction velocity $u *_{t}$ by setting $u *=u_{*}$, we have 


$$
u_{*_{t}}=\frac{\sqrt{1+\left(\sigma^{+} / \bar{u}_{\Delta}^{+}\right)^{2}} \sqrt{\frac{1}{C_{D} L_{D}+C_{L} L_{L}} \frac{\pi d^{2}}{3 S \rho_{f}}\left(\left(\rho_{p}-\rho_{f}\right) L_{w} g d+\frac{C_{C} L_{C}}{d}\right)}}{{\overline{u_{\Delta}}}^{+} \exp \left\{\frac{0.5-p}{|0.5-p|} \sqrt{\ln \left[1+\left(\sigma^{+} / \bar{u}_{\Delta}^{+}\right)^{2}\right]} \sqrt{\frac{\pi}{2} \ln \left[\frac{1}{4 p(1-p)}\right]}\right\}} .
$$

Substituting eq. (29) into $A=\rho_{f} u_{*_{t}}^{2} /\left(\rho_{p}-\rho_{f}\right) g d$, we have

$$
A=f_{p} F_{1} F_{2} G(d)
$$

where

$$
\begin{aligned}
& f_{p}=\frac{L_{w}}{C_{D} L_{D}+C_{L} L_{L}} \frac{\pi d^{2}}{3 S}, \\
& F_{1}=1 /\left({\overline{u_{\Delta}}}^{+}\right)^{2}, \\
& F_{2}=\left[1+\left(\sigma^{+}\right)^{2} F_{1}\right] /\left(\exp \left\{\frac{0.5-p}{|0.5-p|} \sqrt{\ln \left[1+\left(\sigma^{+}\right)^{2} F_{1}\right]} \sqrt{\frac{\pi}{2} \ln \left[\frac{1}{4 p(1-p)}\right]}\right\}\right)^{2},
\end{aligned}
$$

and

$$
G(d)=1+\frac{1}{\left(\rho_{p}-\rho_{f}\right) g} \frac{6 C_{C} L_{C}}{\pi L_{w}} \frac{1}{d^{2}},
$$

All flow properties in eqs (28) to (34) are evaluated at the dimensionless reference height $\left(y_{\Delta}^{+}\right)$. In particular, from eqs. (13) and (14), $\bar{u}_{\Delta}^{+}$is a function of $y_{\Delta}^{+}$and roughness Reynolds number $k_{s}^{+}$, and from eq. (20), $\sigma^{+}$is a function of $y_{\Delta}^{+}$and the flow Reynolds number $\operatorname{Re}_{\tau}$. However, at the threshold of grain motion, both $y_{\Delta}^{+}$and $k_{s}^{+}$are directly proportional to the threshold particle Reynolds number $\mathrm{Re}_{*_{t}}$. Hence eq. (30) replaces eqs. (1) and (2) with the more general formulation

$$
A=f_{p} F_{1}\left(\mathrm{Re}_{*_{t}}\right) F_{2}\left(\mathrm{Re}_{*_{t}}, \operatorname{Re}_{\tau}\right) G(d) .
$$

Eq. (35) states that the dimensionless threshold shear stress $A$ is governed by four types of effect:

i) particle packing geometry, represented by the parameter $f_{p}$;

ii) the mean velocity profile (first-order flow effects), represented by $F_{1}\left(\operatorname{Re}_{*}\right)$;

iii) interaction between the mean velocity profile, turbulent fluctuations and particle removal probability $p$ (second-order flow effects), represented by $F_{2}\left(\operatorname{Re}_{*}, \operatorname{Re}_{\tau}\right)$; and

iv) inter-particle cohesion, represented by $G(d)$. 
Ignoring the turbulent fluctuations (i.e. $\sigma^{+}=0$ and therefore $F_{2}=1$ ) and inter-particle cohesion (i.e. $C_{C} \rightarrow 0$ and therefore $G(d) \rightarrow 1$ ), we have $A \cong f_{p} F_{1}$. In this case, $F_{1}$ can be interpreted as a firstorder effect of the flow that depends solely on the mean velocity profile ${\overline{u_{\Delta}}}^{+}$, which, in turn, depends only on $\operatorname{Re}_{*}$. For similar reasons, $F_{2}$ can be viewed as a second-order effect of the flow as it depends on the turbulent fluctuation $\sigma^{+}$. If we assume $A_{l}=f_{p}$ (see eq. (2)), the main contribution of our new expression (35) is to introduce $F_{2}$, and further specify $F_{1}$ and $G(d)$. Note that the particle packing parameter $f_{p}$ depends on the drag and lift coefficients $C_{D}$ and $C_{L}$, which both depend on the choice of reference height $y$ and $\mathrm{Re}_{*}{ }_{*}$. Such dependences could be important in determining $A$ and its variations. Certain aspects of the statistical effects of particle packing geometry have been discussed by Wu and Chou [2003] and Papanicolaou et al. [2002] and further research is needed to understand the effects of $C_{D}$ and $C_{L}$ in relation to $\operatorname{Re} *{ }_{*}$.

In this paper we focus on the effects of turbulent fluctuations on $A$. The statistical effect of the packing condition will not be considered, and $f_{p}$ is treated as a constant, assuming a fixed packing geometry (Figure 2). Using parameter values defined in Section 2.1, we have $f_{p} \approx 1$ to 2 . For simplicity, an average value of 1.5 is used throughout. In reality, $F_{1}$ and $F_{2}$ may also depend on particle packing conditions, but such effects are also excluded from this paper.

Several expressions have been proposed for the effect of inter-particle cohesion in the past. In general, they share a form similar to

$$
G(d)=1+\frac{K_{1}}{\left(\rho_{p}-\rho_{f}\right) g d^{3-n}},
$$

where $K_{1}$ and $n$ are parameters which need to be calibrated against data. With $n=1$ and $K_{1}=$ $\frac{6 C_{C} L_{C}}{\pi L_{w}}$, eq. (36) reduces to eq. (34) and stands for the assumption of direct proportionality between the cohesion force $F_{C}$ and particle size $d$. Values of the parameters $n$ and $K_{1}$ from previous researchers are listed in Table 2. The resulting expressions for $G(d)$ are plotted in Figure 6 . This shows that inter-particle cohesion becomes negligible when $\mathrm{d}>100 \mu \mathrm{m}$ (in air). However, cohesion is important in determining the upward trend in $A$ with decreasing $\operatorname{Re}_{*_{t}}$, at $\operatorname{Re}_{*_{t}}<1$ (Figure 1).

From Table 2 and Figure 6, it can be inferred that it is reasonable (given earlier work) to assume $F_{C}$ proportional to $d$, and that the most likely range of values for the cohesion coefficient $C_{C}$ is between $10^{-6}$ and $10^{-4} \mathrm{~N} \mathrm{~m}^{-1}$. Though the parameter values listed in Table 2 were all calibrated using 
the wind tunnel data of Iversen and White [1982], they are consistent with values of $C_{C}$ found in adhesion studies. For instance, $C_{C}$ was found to be of the order of $10^{-4} \sim 10^{-2} \mathrm{~N} \mathrm{~m}^{-1}$ in air [Corn, 1961] and $10^{-6} \sim 10^{-4} \mathrm{~N} \mathrm{~m}^{-1}$ in water [Zimon, 1982]. Accordingly, the estimates provided in Table 2 may represent the lower bounds of such effects in air.

\section{[[Insert Figure 6 here]]}

Zimon [1982] attributed the different relationships between $F_{C}$ and particle size $d$, resulting in a range of values of $F_{C}$ for a given $d$, to the probabilistic nature of particle removal. The variance of $F_{C}$ is larger for smaller particles, and may also be larger in air relative to water. For instance, for particle sizes in the range 10 to $20 \mu \mathrm{m}$ in air, $F_{C}$ at a particle removal probability of $50 \%$ can be $10^{2}$ to $10^{3}$ times larger than at a small removal probability ( $2 \%$ to $\left.5 \%\right)$, while for particles smaller than $10 \mu \mathrm{m}$, the difference can be as large as $10^{5}$ [see Fig. 1.2 of Zimon, 1982]. Therefore, for small particles, the effect of the cohesion force on the entrainment threshold is not clear, as a large uncertainty and high variance in $A$ are expected in both experimental data and theoretical estimates for particle size smaller than $10 \mu \mathrm{m}$.

\section{Results and Discussion}

In this section we focus on the first- and second-order flow-related effects, $F_{1}$ and $F_{2}$, mainly considering the range $\operatorname{Re}_{*_{t}}>10$ where $G(d) \rightarrow 1$ (i.e. medium to larger particles). In Section 3.3, we compare our results with experimental data under a variety of different removal probabilities $(p)$ and inter-particle cohesion parameter values $\left(C_{C}\right)$. For all calculations, kinematic viscosity $v$ is set to $1.47 \times 10^{-5} \mathrm{~m}^{2} \mathrm{~s}^{-1}$ in air and $1.0 \times 10^{-6} \mathrm{~m}^{2} \mathrm{~s}^{-1}$ in water, particle density $\rho_{p}$ to $2650 \mathrm{~kg} \mathrm{~m}^{-3}$, and fluid density $\rho_{f}$ to $1 \mathrm{~kg} \mathrm{~m}^{-3}$ in air and $1000 \mathrm{~kg} \mathrm{~m}^{-3}$ in water.

\subsection{First-Order Effects - Effects of the Mean Velocity Profile}

Figure 7 shows contour plots of $F_{1}$ in $k_{s}^{+} \sim y_{\Delta}^{+}$space (dashed lines), with equations accounting for all three flow regimes (eqs. (13), (14) and (15)) in Figure 7a, and for the fully-rough regime only (eqs. (16) and (19)) in Figure 7b. In general, these diagrams show that contour lines of $F_{1}$ are parallel to lines $k_{s}^{+}=\zeta y_{\Delta}^{+}$, where $\zeta$ is a constant. $F_{1}$ reaches a maximum value of round 0.2 at $\zeta=$ 30. When $\zeta<30, F_{1}$ decreases with $y^{+}$but increases with the roughness Reynolds number $k_{s}^{+}$, whereas when $\zeta>30, F_{1}$ increases with $y^{+}$but decreases with $k_{s}^{+}$. In the region where $\zeta<30$, the opposite effects of $k_{s}^{+}$and $y_{\Delta}^{+}$on $F_{1}$ (and therefore on the dimensionless shear stress $A$ ) are the main 
reason that values of $A$ are confined to a rather narrow range in both air and water. The region where $\zeta>30$ may have little to do with threshold entrainment. These points should become increasingly clear later in this Section.

\section{[[Insert Figure 7 here]]}

The differences in $F_{1}$ between Figures 7 a (using all three flow regimes) and Figure $7 \mathrm{~b}$ (using only the fully-rough regime) are most evident when $k_{s}^{+} \leq 70$, where the flow is either smooth or transitionally rough. This is consistent with the findings of Ligrani and Moffat [1986] and Raupach et al. [1991]. In the smooth flow regime, values of $F_{1}$ are larger if the flow is treated as smooth rather than as fully rough. However, in the transitionally-rough regime, values of $F_{1}$ are smaller if the flow is treated as transitionally rough rather as fully rough. Therefore, $A$ can be underestimated for small particles but overestimated for medium particles if fully-rough flow is assumed for all particle sizes. This implies that under/over-estimated values of $A$ can arise as a result of the assumption of fully-rough flow, and explains why the experimental Shields' curve (Figure 1) dips in the vicinity of $\mathrm{Re}_{*_{t}} \sim 10$ to 30 . This is most clearly seen in the data for water. Iversen et al. [1987] stated there is no indication of such a "dip" in $A$ with $\mathrm{Re}_{*_{t}}$ for their own and others' data in air. This may be because fully rough flow is assumed for all atmospheric boundary layer studies [Jiménez, 2004], including the wind tunnel studies of Iversen and his colleagues.

In terms of threshold entrainment, the ratio between the equivalent sand roughness $k_{s}$ and the particle diameter $d$, namely $\alpha=k_{s} / d$, has been discussed by Ling [1995], who indicated that $\alpha$ may be interpreted as a measure of the packing density of roughness elements. For threshold conditions, $\alpha=0.3$ to 4 [Ling, 1995, Cheng and Chiew, 1998], implying that $k_{s}^{+}=\alpha \mathrm{Re}_{*_{t}}$. Commonly used values of $\alpha$ are 1 for uniformly-sized particles and 2 for mixed particle sizes [ $W u$ and Lin, 2002; Wu and Chou, 2003]. On the other hand, according to Section 2.1, $y=\beta d$, implying $y_{\Delta}^{+}=\beta \mathrm{Re}_{*_{t}}$, where $\beta$ lies between 0.5 and 1 . We then have $k_{s}^{+}=\zeta y_{\Delta}^{+}$, where $\zeta=\alpha / \beta$ varies approximately between 0.15 and 4. Larger values of $\zeta$ are possible for other types of packing geometry, as the packing geometry shown in Figure 2 represents the most exposed position for a sphere resting on a surface. Nevertheless, the contour lines outside of $\zeta=0.1$ and $\zeta=10$ may have little relevance for threshold entrainment.

Some typical cases of threshold entrainment are shown in Figure 7. They are $\zeta=0.5,1,2$ and 4, respectively (solid lines). The upper bound of particle size common in gravelly fluvial systems is a grain size of about $100 \mathrm{~mm}$ [Graf, 1971; Buffington and Montgomery, 1997], whereas it is two 
orders of magnitude smaller in aeolian systems [Bagnold, 1941; Greeley and Iversen, 1985]. Thus, the approximate upper bounds of $k_{s}^{+}$and $y_{\Delta}^{+}$are of the order of $10^{4}$ for fluvial systems (the lightlyshaded area), and approximately $10^{2}$ for aeolian systems (dark shading), respectively. This shows that, in air, threshold particle motion is likely to occur within the transitionally-rough and smooth regimes, rather than in the fully-rough regime. It also shows that, for $\zeta=0.5 \sim 4$, the values of $F_{1}$ are mainly limited to $0.01 \sim 0.05$. Multiplying these values by $f_{\mathrm{p}}=1.5$ gives values of $A$ as expected for fluvial entrainment [Graf, 1971; Qian and Wan, 1983; Buffington and Montgomery, 1997], but slightly larger than those obtained in wind tunnels [Bagnold, 1941; Iversen and White, 1982]. The physical cause of such differences will be investigated further in Section 3.2.

In comparison to uniformly-sized beds, $\alpha$ tends to become larger and $\beta$ tends to become smaller for mixed particle beds due to the hiding effect. As has been assumed previously by other researchers, for a given average bed particle size, $\zeta=0.5 \sim 1$ represents more uniformly-sized beds, and a larger $\zeta$ represents mixed-sized surfaces [Wu and Lin, 2002; Wu and Chou, 2003]. Figure 7 provides a theoretical explanation for the observation that $A$ for mixed particle sizes is often larger than that for uniformly sized particles [Shields, 1936; Buffington and Montgomery, 1997]. In addition, as particle sorting processes cause both $\alpha$ and $\beta$ to vary spatially and temporally, they inevitably increase the variations of $A$ [Church, 1978; Andrews, 1983]. This explains why the "dip" in $A$ disappears in data obtained from mixed-sized river beds.

\subsection{Second-Order Effects - Effects of Boundary Layer Friction Reynolds Number $R_{\tau}$}

The mean streamwise velocity is not the only factor affecting threshold entrainment. The turbulent velocity is also responsible for the initial dislodgement of sediment. Eq. (33) provides us the means to investigate the second-order effects on $A$ due to turbulent fluctuations, through the effects of bulk Reynolds number and its relation to particle removal probability. In order to understand the general effect of turbulent fluctuations on $A$, and for simplicity, $\sigma^{+}=\sigma_{\max }^{+}$is assumed at the height of the mean threshold velocity $\overline{u_{\Delta}}$ and is applied to all particle sizes. Eq. (21) is used to relate the maximum turbulent fluctuation $\sigma_{\max }^{+}$to $\operatorname{Re}_{\tau}$.

\section{[[Insert Figure 8 here]]}

Figure 8 shows contour plots of $F_{2}$ in $\sigma^{+} \sim p$ space with $F_{1}$ set to $0.01,0.03$ and 0.06 (left to right panels, respectively). For all three cases, $F_{2}$ increases with removal probability $p$. This shows that, for any given $\operatorname{Re}_{\tau}, F_{2}$ has values smaller than 1 for $0<p<0.3$ and larger than 1 for $0.3<p<1$. Thus, $F_{2}$ acts to increase the variation of $A$. If we assume that $A=f_{p} F_{1}$ (as analyzed in Section 3.1) 
represents the mean, multiplying this quantity by $F_{2}$ reduces the actual value of $A$ when $0<p<0.3$ but enlarges it when $0.3<p<1$.

Figure 8 also shows that $F_{2}$ increases gradually with $\operatorname{Re}_{\tau}$ when $0<p<0.3$, and that this increase is more noticeable for smaller values of $F_{1}$ than for larger ones. Physically, we would expect this to happen as the larger the turbulent fluctuations, the easier the initial dislodgement of sediment, and therefore, the greater the amount of reduction in $A$ in comparison to its mean. We argue that this represents another reason for smaller values of $A$ in air than those obtained in water (Figure 1). According to Figure 5, at the threshold, we may assume that $\operatorname{Re}_{\tau, w}=500$ in flumes and $\operatorname{Re}_{\tau, a}=$ 50000 in wind tunnels respectively. Figure 9 shows the estimated values of $F_{2, w}, F_{2, a}$ and their ratio $\eta=F_{2, \mathrm{w}} / F_{2, a}$ in relation to $p$, with $F_{1, w}=F_{1, a}=0.01$ and 0.04 , respectively. The slope of the $F_{2} \sim p$ relationship is larger in air than that is in water. The two $F_{2} \sim p$ relationships intersect at approximately $p=0.2 \sim 0.3$, and $F_{2}$ in air is about half of its value in water for $p=0.01 \sim 0.05$. The phenomenon that particle entrainment in air is easier for a smaller removal probability, but progressively harder for a larger removal probability for the same particle size entrained by water has also been found experimentally by Zimon [1982]. However, the explanation provided by Zimon [1982] was based upon the probabilistic nature of particle cohesion rather than statistical properties of near-wall turbulence, as argued here.

\section{[[Insert Figure 9 here]]}

Figure 1 suggests that the difference in $A$ between air and water is actually larger than that predicted in Figure 9. This can be attributed to the following reasons. Firstly, it is because of different definitions of particle removal probability at the threshold. Particle removal probabilities at the threshold are never clearly defined under experimental conditions. For instance, those values of $A$ lower than 0.04 were obtained by visual observations, in comparison to those obtained using bedload transport rates [Buffington and Montgomery, 1997]. Secondly, it is shown in Section 3.1 that the average $F_{1}$ may be larger in water than in air. In fact, data reported in Figure 1 are mostly averaged from different experiments with similar conditions. If we assume $F_{1, w}=2 F_{1, a}, f_{p, w}=f_{p, w}$ and $G_{a}(d)=G_{w}(d)=1$ (applicable for medium to large particles), then $A_{w}$ would be approximately 3 to 4 times larger than $A_{a}$ at $p=0.02$. Such a ratio is close to what is seen in Figure 1 .

Previous analyses in hydraulic literature treat the instantaneous pick-up velocity $u_{t}$ and its fluctuation $\sigma$ as linearly proportionality to the friction velocity $u *$, i.e. $u_{t}=C_{t} u_{*}$ and $\sigma=C_{\sigma} u_{*}$, where $C_{t}$ and $C_{\sigma}$ are constants with values of approximately 5.5 and 2.0, respectively [Kironoto and Graf, 1994; Cheng and Chiew, 1998; Wu and Lin, 2002]. Also, fully-rough turbulent flow is 
assumed with no allowance for variations in $\sigma$. A constant value of $\sigma$ fails to predict the systematically lower values of $A$ at large $\operatorname{Re}_{*}{ }_{t}$ observed in air, in comparison to those observed in open channel flow. In this study, $\sigma$ is allowed to vary with bulk Reynolds number as expressed by eqs. (20) and (21). Therefore, linking incipient motion to the scaling of turbulent fluctuation $\sigma$ is the key contribution of this study as this provides us with a fundamental basis to explain the systematically lower values of $A$ in air than in water.

The smaller values of $A$ for smaller removal probabilities in air (and larger values for larger probabilities) suggest that the variance of threshold velocity is larger in air than it is in water. This may have profound implications for understanding wind erosion and aeolian transport. For instance, wind tunnel studies of Shao et al. [1993] found that dust emission is mainly due to saltation bombardment, and that dust emission arising from direct aerodynamic entrainment is generally small. On the other hand, direct field observations of Loosmore and Hunt [2000] found dust suspension can be initialized by turbulent eddies in surface winds well before the sandblasting mechanism. Similar observations were made by Roney and White [2004] at Owens Lake. Both Loosmore and Hunt [2000] and Roney and White [2004] found that the threshold velocity is smaller than the values estimated by the conventional "saltation thresholds". From Figure 8 and 9, we suggest that the physical causes of the discrepancy are likely to be due to the different definitions of the threshold removal probability. While Shao et al. [1993] looked at sustained dust emission rates at an average $p$ (at approximately $30 \%$ to $50 \%$ ), the latter two studies focused on initial uplifting at $p=1 \sim 10 \%$.

Figures 7 and 8 also show that $F_{1}$ may also affect $F_{2}$. At smaller $p$, the effect of $\operatorname{Re}_{\tau}$ on $A$ becomes less evident when $F_{1}$ becomes larger. Also, for a given $p, F_{2}$ increases with $F_{1}$. Physically, this means that when $A$ is larger, there is less reduction in its value by the second-order effect (i.e. when multiplying it by $F_{2}$ ). Further experimental studies are needed to investigate these findings. In reality, the effects of $\operatorname{Re}_{\tau}$ and $\operatorname{Re}_{*_{t}}$ are more complex than those presented in Figures 7 and 8 . This is because $A, \operatorname{Re}_{\tau}$ and $\operatorname{Re}_{*}$ are all related to threshold velocity $u_{*}$, which increases with particle size. In addition, boundary layer height $\delta$ may change with particle size.

Direct field measurements by Roney and White [2004] at Owens Lake found that the dust suspension threshold varied between 50 and $75 \%$ of the values estimated from expressions derived from wind tunnel experiments. The general model proposed in this study provides the physical explanation for such an observation. Figure 10 shows three possible $A \sim \operatorname{Re}_{{ }^{*}}$ relationships at boundary layer heights of $\delta=0.01 \mathrm{~m}, 1 \mathrm{~m}$, and $1000 \mathrm{~m}$, respectively, all for $p=0.05$. These curves were obtained, for an in-air situation, by substituting eqs. (13) and (20) in eqs. (32) and (33) with 
$y_{\Delta}^{+}=0.6 \mathrm{Re}_{*}, k_{s}^{+}=\mathrm{Re}_{*_{t}}$ and $C_{C}=4 \times 10^{-5} \mathrm{~N} \mathrm{~m}^{-1}$. The values of $A$ were calculated through iteration over $u *_{t}$ for a given particle size $d$. This shows that $A$ decreases with $\delta$ and the decrement increases when $\mathrm{Re}_{*}$ increases, suggesting that, for the same values of $\mathrm{Re}_{*}$, direct field measurement would result in smaller $A$ in comparison to that in wind tunnel conditions. The physical implication is that, for a given particle size, the average threshold velocity for dislodging particles could be smaller in the atmospheric boundary layer compared with that observed in wind tunnels. Alternatively, a particle that remains still in a wind tunnel may move if it is in an atmospheric boundary layer at the same wind velocity.

\section{[[Insert Figure 10 here]]}

\subsection{Comparison with Experimental Data}

In this section, using an approach similar to that employed to derive Figure 10, we shall use eqs. (13) and (20) to estimate $F_{1}$ and $F_{2} . A$ is then calculated through iteration over $u *_{t}$ for a given particle size $d$. For all calculations below, we assume $y_{\Delta}^{+}=0.6 \mathrm{Re}_{*}$. When comparing predictions with data from flume experiments, we assume $k_{s}^{+}=3 \mathrm{Re}_{*_{t}}$ and $\delta=0.02 \mathrm{~m}$, while $k_{s}^{+}=\mathrm{Re}_{*_{t}}$ and $\delta=1.2 \mathrm{~m}$ for comparing with data from wind tunnels.

Figure 11 shows predicted $A \sim \mathrm{Re}_{*}{ }_{t}$ relationships for four values of $p$ in air and in water and three values of the cohesion coefficient $C_{C}$, respectively. For a given fluid, the variation of $A$ in relation to $\operatorname{Re}_{*}$ can be explained by differences in the removal probability $p$ when $\operatorname{Re}_{*_{t}}>10$, by interparticle cohesion when $\operatorname{Re}_{*_{t}}<1$, and by both of them when $\operatorname{Re}_{*_{t}}$ is in between. The Figure shows that, with a mean value of $C_{C}=10^{-5} \sim 10^{-4} \mathrm{~N} \mathrm{~m}^{-1}$, the new expression agrees very well with experimental data for both air and water. The $C_{C}$ values used in this study are smaller than those suggested previously [Shao and Lu, 2000; Cornelis and Gabriels, 2004], but this is because flowrelated effects play an increasingly important role in the new expression we propose. As discussed in Section 3.1 and shown in Figure 7, at smaller $k_{s}^{+}$and $y_{\Delta}^{+}$(therefore smaller $\operatorname{Re} *_{t}$ ), $F_{1}$ (therefore $A$ ) increases with $\operatorname{Re}_{*}$.

\section{[[Insert Figure 11 here]]}

Previous researchers have suggested that the particle-to-fluid density ratio $R_{\rho}=\left(\rho_{p}-\rho_{f}\right) / \rho_{f}$ is an important parameter in determining $A$ [Iversen et al., 1987; Cornelis and Gabriels, 2004]. Our new expression suggests that $A$ also depends on both $\operatorname{Re}_{*}$ and $\operatorname{Re}_{\tau}$, not just on $R_{\rho}$. Therefore, in terms of the effects of flow on $A$, not only the density but also the flow viscosity is important. For a set of 
fixed values of viscosity, particle size, and boundary layer height, our new expression suggests that $A$ increases when $R_{\rho}$ decreases, as was shown by the wind tunnel data of Iversen and White [1982]. However, it would no longer be these parameters also vary, according to our generalized model. The experimental simulations of McKenna Neuman [2003; 2004] confirm that $A$ and sediment transport rates depend not only on fluid density ( or $R_{\rho}$ ) but also on fluid viscosity. She showed both threshold velocity and mass transport rates of sedimentary particles vary with temperature (Selby et $a l ., 1974)$ and humidity. For instance, it was found that the aerodynamic drag required to entrain sand size particles can be $30 \%$ lower under cold conditions in high latitude regions, as compared to hot deserts and mass transport rates increase while temperature decreases. McKenna Neuman [2004] suggested that temperature-dependent changes in air density and viscosity, and turbulence, are the major affecting factors. This example shows that caution is needed in applying previous analytical and semi-empirical models, and in assuming fixed parameter values, as both models and parameters may only be applicable to certain conditions.

Both theoretical analysis and observation show a certain degree of variation in $A$. We therefore argue that threshold entrainment should be viewed statistically rather than deterministically, as most governing processes are probabilistic in nature, including near-surface turbulent flow, packing geometry [Kirchner et al., 1990; Papanicolaou et al., 2002] and inter-particle cohesion. For instance, the most important process - rough wall turbulent motion - is fundamentally stochastic. The structure of near-bed turbulent flow is characterized by the spatial and temporal organization of coherent structures [Best, 1992], the links between outer and inner scales, the nature of intermittency and the role of anisotropy. All these turbulence characteristics depend on and interact with the bed condition in a complex manner. In addition, for small values of $\mathrm{Re}_{*}$, the interparticle cohesion forces are also statistical in nature, and the relationships between cohesion forces and particle size are very complex and not well understood (see Section 2.1). For all these reasons, a deterministic resolution of incipient motion may not be possible. This message is particularly relevant to wind erosion studies where a deterministic view of incipient motion remains common, and data on incipient motion are too sparse to separate the variety of physical processes involved.

\section{Conclusions}

In this study, we have proposed a new, general expression for the dimensionless threshold shear stress $A$ which is applicable to both aeolian and fluvial particle entrainment. It allows us to study the effects of mean flow, turbulent fluctuation, and inter-particle cohesion on incipient motion separately. It suggests that the dimensionless threshold shear stress $A$ is indeed related to $\mathrm{Re}_{*}$, as 
earlier researchers proposed, and should also depend on flow Reynolds number $\operatorname{Re}_{\tau}$, which is proposed for the first time. Therefore, $A$ depends on the flow condition and it should be a function of the viscosity (a flow property) as well as being a function of the density ratio and particle size. We have shown that the differences in the probability distribution of streamwise velocity fluctuations for typical situations in air and water are the main reason for the larger values of $A$ in water than in air (Figure 1). This is because the turbulent fluctuations are not only related to the near-bed flow structure (i.e. $\mathrm{Re}_{*_{t}}$ ), as expected, but also depend on the bulk flow characteristics (i.e. $\mathrm{Re}_{\tau}$ ). As velocity variance increases with the bulk flow Reynolds number $\operatorname{Re}_{\tau}$, and typical values of $\operatorname{Re}_{\tau}$ in air are several orders larger than those in water under the conditions of incipient motion, so the values of $A$ are smaller in air than in water. The upturn of $A$ for small $\operatorname{Re}_{*}{ }_{t}$ is partly due to the flow conditions and partly due to inter-particle cohesion. Therefore, incorporating descriptions of the flow condition into threshold entrainment is indispensable for all particle sizes.

At large $\mathrm{Re}_{*}$, the narrow range of values of $A$ in relation to $\mathrm{Re}_{*_{t}}$ results from the opposite effects of the roughness Reynolds number $k_{s}^{+}$and the dimensionless reference height $y_{\Delta}^{+}$, both of which are proportional to the particle Reynolds number $\mathrm{Re}_{*_{t}}$. Although the mean values of $A$ may be within a narrow range, certain systematic variations exist. These variations are due to differences in particle packing density, which is incorporated in the first-order effect $F_{1}$; and to the different removal probability $(p)$ used in different experiments, and the bulk Reynolds number, which are incorporated in the second-order effect $F_{2}$. In reality, these effects are likely to act interactively on $A$ and are reflected in a combined but rather random manner in experimental studies.

\section{ACKNOWLEDGEMENTS}

HL is grateful to Professor Herbert Huppert at The Institute of Theoretical Physics, University of Cambridge, for his kind support. We would like to thank two anonymous reviewers for their thoughtful comments, which greatly improved this paper. 


\section{References}

Andrews, E. D., Entrainment of gravel from naturally sorted riverbed material, Geol. Soc. Am. Bull., 94, 1225-1231, 1983.

Bagnold, R. A., The physics of blown sand and desert dunes, Methuen, London, 1941.

Bagnold, R. A., The flow of cohesionless grains in fluids, Philosophical Trans. Roy. Soc. London, A, 249 No.964, 1956.

Barenblatt, G. I., and A. J. Chorin, Scaling of the intermediate region in wall-bounded turbulence: The power law. Phys. Fluids, 10, 1043-1044, 1998.

Batchelor, G. K., An introduction to fluid dynamics, Cambridge University Press, Cambridge, $615 \mathrm{pp}, 1967$.

Best, J. L., On the entrainment of sediment and initiation of bed defects: insights from recent developments within turbulent boundary layer research. Sedimentology, 39, 797-811, 1992.

Bridge, J. S., and S. J. Bennett, A model for the entrainment and transport of sediment grains of mixed sizes, shapes, and densities, Water Resours. Res., 28(2), 337-363, 1992.

Buffington, J. M., and D. R. Montgomery, A systematic analysis of eight decades of incipient motion studies, with special reference to gravel-beded rivers, Water Resour. Res., 33, 19932029, 1997.

Bergstrom, D. J., M. F. Tachie, and R. Balachandar, Application of power laws to low Reynolds number boundary layers on smooth and rough surfaces, Phys. Fluids, 13(11), 3277-3284, 2001.

Cheng, N. S., and Y. M. Chiew, Pickup probability for sediment entrainment, J. Hydraul. Eng., 124(2), 232-235, 1998.

Chepil, W. S., The use of evenly spaced hemisphere to evaluate aerodynamic forces on a soil surface, Trans. Am. Geophys. Union, 39(3), 397-404, 1958.

Church, M., Palaeohydrological reconstructions from a Holocene valley fill, In: Miall, A. D. (Ed.), Fluvial Sedimentology, Can. Soc. of Pet. Geol., Calgary, Alberta. 743-772, 1978

Clauser, F. H., The turbulent boundary layer, Adv. Appl. Mechs., 4, 1-51, 1956.

Cleaver, J. W., and B. Yates, Mechanism of detachment of colloidal particles from a flat substrate in a turbulent flow, J. Colloid Interface Sci., 44:464-474, 1973. 
Coleman, N. L., A theoretical and experimental study of drag and lift forces acting on a sphere resting on hypothetical stream bed, Proc., $12^{\text {th }}$ compress, IAHR, Fort Collins, Colo., 3, 185$192,1967$.

Corn, M., Adhesion of solid particles to solid surface, I: A review, J. Air Pollution Control Assoc., $11,523-537,1961$.

Cornelis, W. M., and D. Gabriels, A simple model for the prediction of the deflation threshold shear velocity of dry loose particles, Sedimentology, 51, 39-51, 2004.

Einstein, H. A., The bed load function for sediment transportation in open channel flows, Tech. Bull. 1026, USDA, Washington, D.C, 1950.

Fischer, P., G. Leaf, and J. Restrepo, Forces on particles in oscillatory boundary layers, J. Fluid Mech., 468, 327-347, 2002.

Fletcher, B., The erosion of dust by an airflow, J. Phys. D: Appl. Phys., 9, 913-524, 1976a.

Fletcher, B., The incipient motion of granular materials, J. Phys. D: Appl. Phys., 9, 2471-2478, 1976b.

Fuks, N. A., Aerosol Mechanics, 358pp, Nauka, Moscow, 1955.

DeGraaff, D. B., and J. K. Eaton, Reynolds number scaling of the flat plate turbulent boundary layer, J. Fluid Mech., 422, 319-346, 2000.

Gessler, J., Beginning and ceasing of sediment motion, In: H. W. Shen (Ed.), River mechanics, Fort Collins, Colo., 7:1-7:22, 1971.

Graf, W. H., Hydraulics of Sediment Transport, McGraw-Hill Book Company, New York, 1971.

Grass, A. J., Initial instability of fine sand, J. Hydraul. Div., Am. Soc. Civ. Eng., 96(3), 619-632, 1970.

Greeley, R., and J. D. Iversen, Wind as a Geological Process on Earth, Mars, Venus and Titan, Cambridge University Press, New York, 1985.

Iversen, J. D. and B. R. White, Saltation threshold on Earth, Mars and Venus, Sedimentology, 29, 111-119, 1982.

Iversen, J. D., R. Greeley, J. R. Marshall, and J. B. Pollack, Aeolian saltation threshold: the effect of density ratio, Sedimentology, 34, 699-706, 1987.

Jackson, P. S., On the displacement height in the logarithmic velocity profile, J. Fluid Mech., 111, $15-25,1981$. 
James, C., Prediction of entrainment conditions for nonuniform, noncohesive sediments, J. Hydraul. Res. 28(1), 25-41, 1990.

Jiménez, J., Turbulent Flows over Rough Walls, Ann. Rev. Fluid Mech., 36, 173-96, 2004.

Kirchner, J. W., W. E. Dietrich, F. Iseya, and H. Ikeda, The variability of critical shear stress, friction angle, and grain protrusion in water-worked sediments, Sedimentology, 37, 647-672, 1990.

Kironoto, B. A., and W. H. Graf, Turbulence characteristics in rough uniform open-channel flow, Proc. Inst. Civ. Eng., Water, Maritime Energ., 106, 333-344, 1994.

Komar, P.D. and Li, Z., Applications of grain-pivoting and sliding analyses to selective entrainment of gravel and flow competence evaluations. Sedimentology, 35, 681-695.

Krogstad, P. A., and R. A. Antonia, Surface roughness effects in turbulent boundary layers, Exp. Fluids, 27, 450-460, 1999.

Ligrani, P. M., and R. J. Moffat, Structure of transitionally rough and fully rough turbulent boundary layers, J. Fluid Mech., 162, 69-98, 1986.

Ling, C. H., Criteria for incipient motion of spherical sediment particles, J. Hydraul. Eng., 121(6), 47-478, 1995.

Loosmore, G. A., and J. R. Hunt, Below-threshold, non-abraded dust resuspension, J. Geophy. Res., 105, 20663-20671, 2000.

Mantz, P., Incipient transport of fine grains and flakes by fluids - Extended Shields diagram, $J$. Hydraul. Div. Am. Soc. Civ. Eng., 103(6), 601-615, 1977.

Marsh, N. A., A.W. Western, and R. B. Grayson, Comparison of methods for predicting incipient motion for sand beds, J. Hydraul. Eng., 130(7), 616-621, 2004.

Marusic, I., and G. J. Kunkel, Streamwise turbulence intensity formulation for flat-plate boundary layers, Phys. Fluids, 15(8), 2461-2464, 2003.

McKenna Neuman, C., Effects of temperature and humidity upon the entrainment of sedimentary particles by wind, Boundary-Layer Meteorology, 108, 61-89, 2003.

McKenna Neuman, C., Effects of temperature and humidity upon the transport of sedimentary particles by wind, Sedimentology, 51, 1-18, 2004.

Metzger, M., J. Klewicki, K. Bradshaw, and R. Sadr, Scaling the near-wall axial turbulent stress in the zero pressure gradient boundary layer, Physics of Fluids, 13, 1819-1821, 2001. 
Morrison, J. F., B. J. McKeon, W. Jiang, and A. J. Smits, Scaling of the streamwise velocity component in turbulent pipe flow, J. Fluid Mech., 508, 99-131, 2004.

Nickling, W. G., The initiation of particle movement by wind, Sedimentology, 35, 499-511, 1988.

Nikuradse, J., Strömungsgesetze in Rauhen Rohren, VDI-Forsch. 361, 1933 (Engl. transl. 1950. Laws of flow in rough pipes, NACA TM 1292).

Panton, R., Review of wall turbulence as described by composite expansions, Applied Mechanics Reviews, Trans. ASME, 58, 1-36, 2005.

Papanicolaou, A., P. Diplas, C. L. Dancey, and M. Balakrishnan, Surface roughness effects in nearbed turbulence: Implications to sediment entrainment, J. Eng. Mech., 127(3), 211-218, 2001.

Papanicolaou, A. N., P. Diplas, N. Evaggelopoulos, and S. Fotopoulos, Stochastic incipient motion criterion for spheres under various bed packing conditions, J. Hydraul. Eng., 128(4), 369$380,2002$.

Patnaik, P. C., N. Vittal, and P. K. Pande, Lift coefficient of a stationary sphere in gradient flow, J. Hydraul. Res., 32(3), 471-480, 1994.

Phillips, M., A force balance model for particle entrainment into a fluid stream, J. Phys. D: Appl. Phys., 13, 221-233, 1980.

Qian, N., and C. H. Wan, Mechanics of sediment transport, Science press, Beijing (in Chinese), 1983 (Engl. Transl. by J. S. McNown, 1998, American Society of Civil Engineers).

Raupach, M. R., Drag and drag partition on rough surfaces, Boundary-Layer Meteorol., 60, 375-395, 1992.

Raupach, M. R., R. A. Antonia, and S. Rajagopalan, Rough-Wall Turbulent Boundary Layers, Appl. Mech. Rev., 44, 1-25, 1991.

Raupach, M. R., and H. Lu, Representation of land-surface processes in Aeolian transport models, Environ. Modelling \& Software, 19, 93-112, 2004.

Raupach, M.R., J.J. Finnigan and Y. Brunet, Coherent eddies and turbulence in vegetation canopies: the mixing layer analogy, Boundary-Layer Meteorol. 78, 351-382, 1996.

Richards, K.S., Fluvial geomorphology: initial entrainment of bed material in gravel-bed rivers. Progress in Physical Geography, 14, 395-415, 1990.

Roney, J. A., and B. R. White, Definition and measurement of dust aeolain thresholds, J. Geophys. Res., 109, F01013, doi: 10.1029/2003KF000061, 2004. 
Selby, M. J., R. B. Rains, and R. W. P. Palmer, Eolian deposits of the ice-free Victoria Valley, Southern Victoria Land and Antarctica. New Zealand Journal of Geology and Geophysics, 17(3), 543-562, 1974

Shao, Y., and H. Lu, A simple expression for wind erosion threshold friction velocity, J. Geophy. Res., 105, 22437-22443, 2000.

Shao, Y., M. R. Raupach, and P. A. Findlater, The effect of saltation bombardment on the entrainment of dust by wind, J. Geophy. Res., 98, 12719-13726, 1993.

Shields, A., Application of similarity principles and turbulence research to bed-load movement (in German), Preuss. Vers. Anst. Wasserb. Schiffb., No. 26, Berlin, 1936.

Tachie, M. F., D. J. Bergstrom, and R. Balachandar, Roughness effects on the mixing properties in open channel turbulent boundary layers, J. Fluids Eng., 126, 1025-1032, 2004.

Theodoor, J., and G. Overbeek, Birth, life and death of colloids, In: Eicke, E. H.-F. (Ed.), Modern Trends in Colloid Science in Chemistry and Biology, 9-33. Springer-Verlag, 1985.

Tillman, W., Neue Widerstandsmessungen an Oberflächenstörunden in der turbulenten Reibungsschicht, ZWB Untersuch. Mitteil. 6619, 1944 (Engl. Transl. 1951, Additional measurements of the drag of surface irregularities in turbulent boundary layers. NACA TM 1299).

Wu, F. C., and Y. J. Chou, Rolling and lifting probabilities of sediment entrainment, J. Hydraul. Eng., 129(2), 110-119, 2003.

Wu, F. C., and Y. C. Lin, Pickup probability of sediment under Log-normal velocity distribution, $J$. Hydraul. Eng., 128(4), 438-442, 2002.

White, S. J., Plane bed thresholds of fine grained sediments, Nature, 228, 152-153, 1970.

Yalin, M. S., Mechanics of sediment transport, Pergamon Press, Oxford, 1972.

Yalin, M. S., and E. Karahan, Inception of sediment transport, J. Hydraul. Div., Am. Soc. Civ. Eng., 105, 1433-1443, 1979.

Zimon, D. Z., Adhesion of Dust and Powder, Translated by R. K. Johnston, Consultants Bureau, Plenum Press, New York, 1982.

Zingg, A.W., Wind tunnel studies of the movement of sedimentary material, Proceedings 5th Hydraulic Conference Bulletin, 34, 111-35, 1953. 
Table 1. Main symbols (omitting symbols used only once)

\begin{tabular}{|c|c|c|}
\hline Symbol & Definition & First use \\
\hline$A$ & dimensionless threshold shear stress, $A=\rho_{f} u_{*_{t}}^{2} /\left(\left(\rho_{p}-\rho_{f}\right) g d\right)$ & $\mathrm{Eq}(1)$ \\
\hline$A_{1}$ & reference value of $A$ & $\mathrm{Eq}(2)$ \\
\hline$B$ & $\begin{array}{l}\text { empirical constant in logarithmic law over a smooth wall } \\
(B=5.0)\end{array}$ & $\mathrm{Eq}(12)$ \\
\hline$c_{i}$ & cohesion coefficient & $\mathrm{Eq}(6)$ \\
\hline$C_{D}, C_{L}$ & drag, lift coefficients (computations assume $C_{D}=0.5, C_{L}=0.3$ ) & Eqs (4), (5) \\
\hline$d$ & particle diameter & $\mathrm{Eq}(1)$ \\
\hline $\begin{array}{l}F_{D}, F_{L} \\
F_{G}, F_{C}\end{array}$ & forces on particle from drag, lift, specific weight and cohesion & Eqs (3) to (5) \\
\hline$F\left(\operatorname{Re}_{*}\right)$ & $\begin{array}{l}\text { dimensionless function quantifying dependence of } A \text { on } \\
\text { gravitational and aerodynamic forces }\end{array}$ & $\mathrm{Eq}(2)$ \\
\hline$\overline{f_{p}}$ & $\begin{array}{l}\text { component of } F \text { associated with packing geometry (computations } \\
\text { assume } f_{p}=1.5 \text { ) }\end{array}$ & Eq (31) \\
\hline$F_{1}$ & component of $F$ associated with first-order flow effects & $\mathrm{Eq}(32)$ \\
\hline$F_{2}$ & component of $F$ associated with second-order flow effects & Eq (33) \\
\hline$G(d)$ & $\begin{array}{l}\text { dimensionless function quantifying dependence of } A \text { on cohesion } \\
\text { forces }\end{array}$ & Eq (2) \\
\hline$g$ & gravitational acceleration & $\mathrm{Eq}(1)$ \\
\hline$k_{s}$ & Nikuradse sand-grain roughness & $\mathrm{Eq}(14)$ \\
\hline$K_{1}$ & dimensional parameter for cohesion force & $\mathrm{Eq}(36)$ \\
\hline $\begin{array}{l}L_{D}, L_{L} \\
L_{G}, L_{C}\end{array}$ & $\begin{array}{l}\text { moment arms for forces } F_{D}, F_{L}, F_{G}, F_{C}\left(L_{D}=d \cos \theta, L_{L}=d \sin \theta,\right. \\
\left.L_{G}=d \sin \theta \text { and } L_{C}=d \sin \theta\right)\end{array}$ & $\mathrm{Eq}(8)$ \\
\hline$n$ & exponent specifying cohesion force & $\mathrm{Eq}(36)$ \\
\hline$p$ & probability of entrainment & $\mathrm{Eq}(23)$ \\
\hline $\operatorname{Re}_{\tau}$ & flow Reynolds number, $\operatorname{Re}_{\tau}=u_{*} \delta / \nu$ & Sec 2.3 \\
\hline $\mathrm{Re}_{*}$ & particle Reynolds number at threshold, $\mathrm{Re}_{*_{t}}=u_{*_{t}} d / v$ & Eq (1) \\
\hline$S$ & $\begin{array}{l}\text { horizontal area of particle exposed to the flow (computations } \\
\text { assume } S=0.2 \pi d^{2} \text { ) }\end{array}$ & Eqs (4), (5) \\
\hline$u$ & streamwise velocity (dimensionless form: $u^{+}=u / u_{*}$ ) & $\operatorname{Sec} 2$ \\
\hline$u_{\Delta}$ & reference velocity (at height $y_{\Delta}$ ) for defining $F_{D}$ and $F_{L}$ & Eqs (4), (5) \\
\hline$u_{t}$ & threshold velocity (at height $y_{\Delta}$ ) & $\mathrm{Eq}(10)$ \\
\hline$u *_{t}$ & threshold friction velocity & $\mathrm{Eq}(1)$ \\
\hline$v_{\Delta}, v_{t}$ & $v_{\Delta}=\ln u_{\Delta}, v_{t}=\ln u_{t}$ & $\mathrm{Eq}(22)$ \\
\hline$y$ & height above level of effective drag $(y=0)$ & $\operatorname{Sec} 2$ \\
\hline$y^{+}$ & dimensionless height, $y^{+}=u_{*} y / \nu$ & Sec 2 \\
\hline$y_{\Delta}$ & height at which velocity $u_{\Delta}$ is defined & Eqs (4), (5) \\
\hline$y_{\Delta}^{+}$ & dimensionless reference height at which velocity $u_{\Delta}$ is defined & $\operatorname{Sec} 2$ \\
\hline$z_{0}$ & roughness length & $\mathrm{Eq}(16)$ \\
\hline$z_{i}$ & smallest separation between two spherical particles in Eq. (6) & $\mathrm{Eq}(6)$ \\
\hline$\beta$ & constant relating reference height $y_{\Delta}$ to $d$ & Sec 2.1 \\
\hline$\delta$ & boundary-layer depth & Sec 2.3 \\
\hline$\Delta \bar{u}^{+}$ & velocity-increment form of roughness function & $\mathrm{Eq}(13)$ \\
\hline
\end{tabular}




\begin{tabular}{|lll|}
\hline$\kappa$ & von Karman constant $(\kappa=0.41)$ & Eq (12) \\
\hline$v$ & kinematic viscosity of the fluid. & Eq (1) \\
\hline$\xi$ & Interpolation function for roughness in transition regime & Eq (15) \\
\hline$\rho_{f}, \rho_{p}$ & fluid density, particle density & Eq (1) \\
\hline$\theta$ & pivoting angle (see Figure 2; computations assume $\left.\theta=30^{\circ}\right)$ & Eq (8) \\
\hline$\sigma$ & standard deviation of streamwise velocity (dimensionless form: & Eq (20) \\
& $\left.\sigma^{+}=\sigma / u_{*}\right)$ & \\
\hline$\left.\tau_{*}\right)$ & threshold shear stress, $\tau_{*_{t}}=\rho_{f} u_{*_{t}}^{2}$ & Eq (1) \\
\hline
\end{tabular}


Table 2. Parameter values for eq. (36) and implied values of the cohesion parameter $C_{C}$.

\begin{tabular}{l|l|l|l}
\hline \multicolumn{1}{c|}{$K_{1}$} & \multicolumn{1}{|c|}{$C_{C}$} & \multicolumn{1}{c|}{$n$} & \multicolumn{1}{c}{ Researchers } \\
\hline $6 \times 10^{-7} \mathrm{~N} \mathrm{~m}^{-0.5}$ & $3.1 \times 10^{-7} \mathrm{~N} \mathrm{~m}^{-0.5}$ & 1.5 & Greeley and Iversen [1985] \\
$0.055^{*}$ & 0.029 & 1 & Iversen et al. [1987] \\
$1.65 \times 10^{-4}$ to $5.0 \times 10^{-4} \mathrm{~N} \mathrm{~m}^{-1}$ & $8.6 \times 10^{-5}$ to $2.6 \times 10^{-4} \mathrm{~N} \mathrm{~m}^{-1}$ & 1 & Shao and Lu [2000] \\
$1.69 \times 10^{-4}$ to $1.77 \times 10^{-4} \mathrm{~N} \mathrm{~m}^{-1}$ & $8.8 \times 10^{-5}$ to $9.3 \times 10^{-5} \mathrm{~N} \mathrm{~m}^{-1}$ & 1 & Cornelis and Gabriels [2004] \\
$7 \times 10^{-6} \mathrm{~N} \mathrm{~m}^{-1.3}$ & $3.7 \times 10^{-6} \mathrm{~N} \mathrm{~m}^{-1.3}$ & 1.3 & Cornelis and Gabriels [2004] \\
\hline
\end{tabular}

* Note that the unit of $K_{1}$ was not specified in Iversen et al. [1987]. 


\section{Figure Captions}

Figure 1. Dimensionless threshold shear stress $A$ as a function of particle Reynolds number $\operatorname{Re}_{*}$. The diagram shows that data obtained in water flow follow a different trend compared with those obtained in air stream. Data were extracted from the literature indicated in the legend.

Figure 2. Schematic of an erodible particle resting on other similar particles. Forces acting on the shaded particle include the aero/hydro-dynamic drag, $F_{\mathrm{D}}$, the aero/hydro-dynamic lift $F_{\mathrm{L}}$, the gravitational force, $F_{\mathrm{G}}$, and the net cohesive force, $F_{\mathrm{C}}$. The vertical distribution of stream-wise mean velocity $\bar{u}$ is also shown.

Figure 3. Schematic diagram of probability of entrainment $p$ equal to probability of exceedance $\operatorname{Prob}\left(u_{\Delta} \geq u_{t}\right)$, where $u_{\Delta}$ and $u_{t}$ are the instantaneous reference velocity and the threshold velocity, respectively.

Figure 4. Normalized stream-wise turbulence intensities $\sigma^{+}$as a function of normalized vertical height $y^{+}$and boundary layer friction Reynolds number $\operatorname{Re}_{\tau}$. The lines are calculated using eq. (20).

Figure 5. Peak values of $\sigma^{+}$from Figure 4 as a function of $\operatorname{Re}_{\tau}$. Typical ranges of $\operatorname{Re}_{\tau}$ for flumes, natural rivers, wind tunnels and atmospheric boundary layers are also shown.

Figure 6. Previously published relationships of $G(d)$ as a function of particle size $d$.

Figure 7. Contour plots of $F_{1}$ in $y^{+} \sim k_{s}^{+}$space. In (a) eqs. (13) to (15) were used for the calculation; in (b) eq. (16) with $z_{0} / k_{s}=0.031$ was used for the calculation. Note that $y^{+}$is replaced by $y_{\Delta}^{+}$for the threshold condition of grain motion. See text for details.

Figure 8. Contour plot of $F_{2}$ in $\operatorname{Re}_{\tau} \sim p$ space. From left to right, the panels are for $F_{1}=0.01,0.03$, and 0.06 , respectively.

Figure 9. Left panels: $F_{2}$ as a function of $p$ for the cases of air and water for $F_{1}=0.01$ (upper panel) and $F_{1}=0.04$ (lower panel). Right panels: the ratio of $F_{2}$ in water and air for the same $F_{1}$ values as on the left.

Figure 10. Computed $A \sim \mathrm{Re}_{*}{ }_{t}$ relationship in air for three different values of boundary layer height $\delta$.

Figure 11. Comparison between computed $A \sim \operatorname{Re}_{*}{ }_{t}$ relationships in air and water. Dots shown are experimental data as in Figure 1, complemented by other data obtained from flume experiments [Buffington and Montgomery, 1997]. 


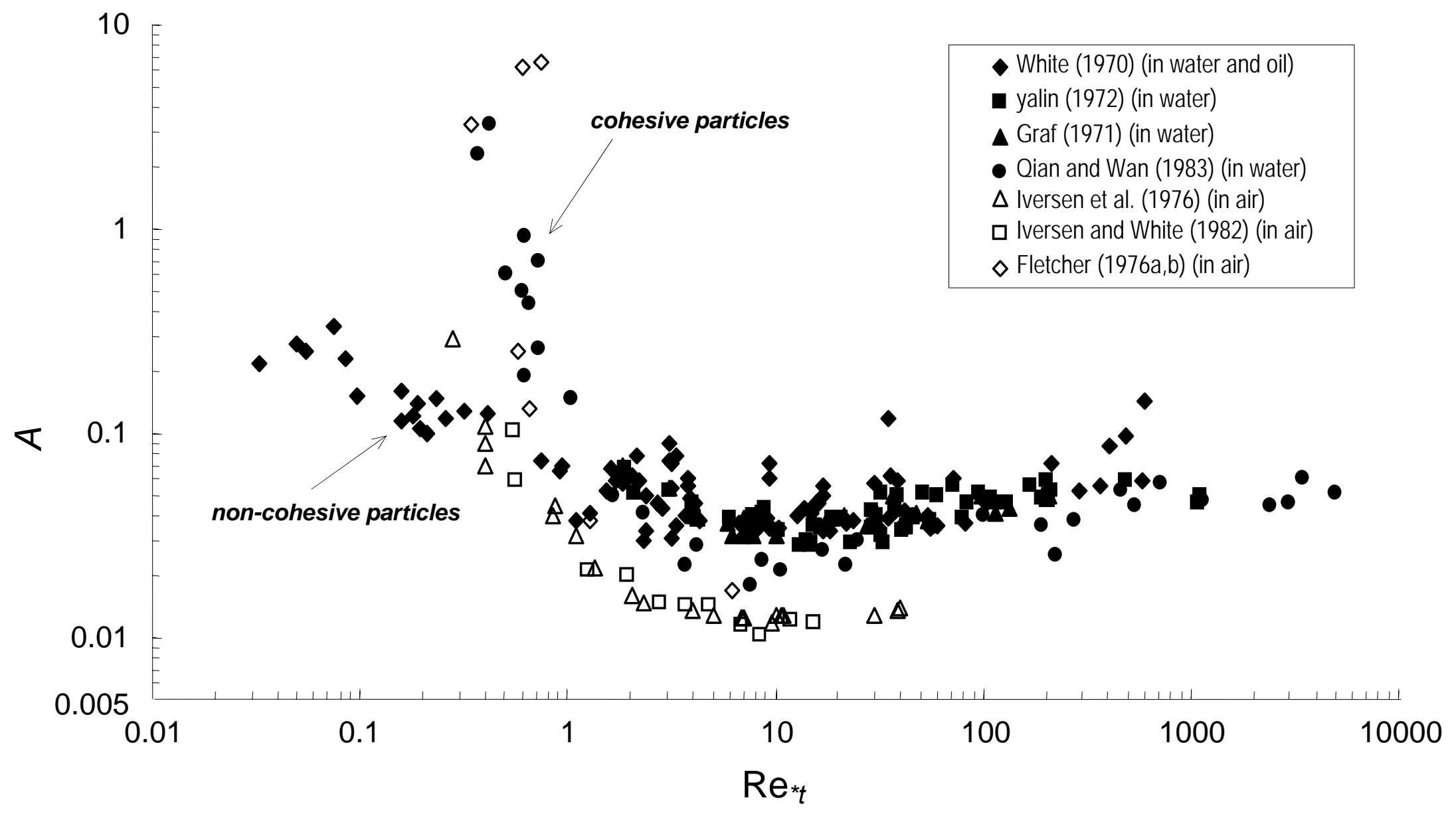

Figure 1 


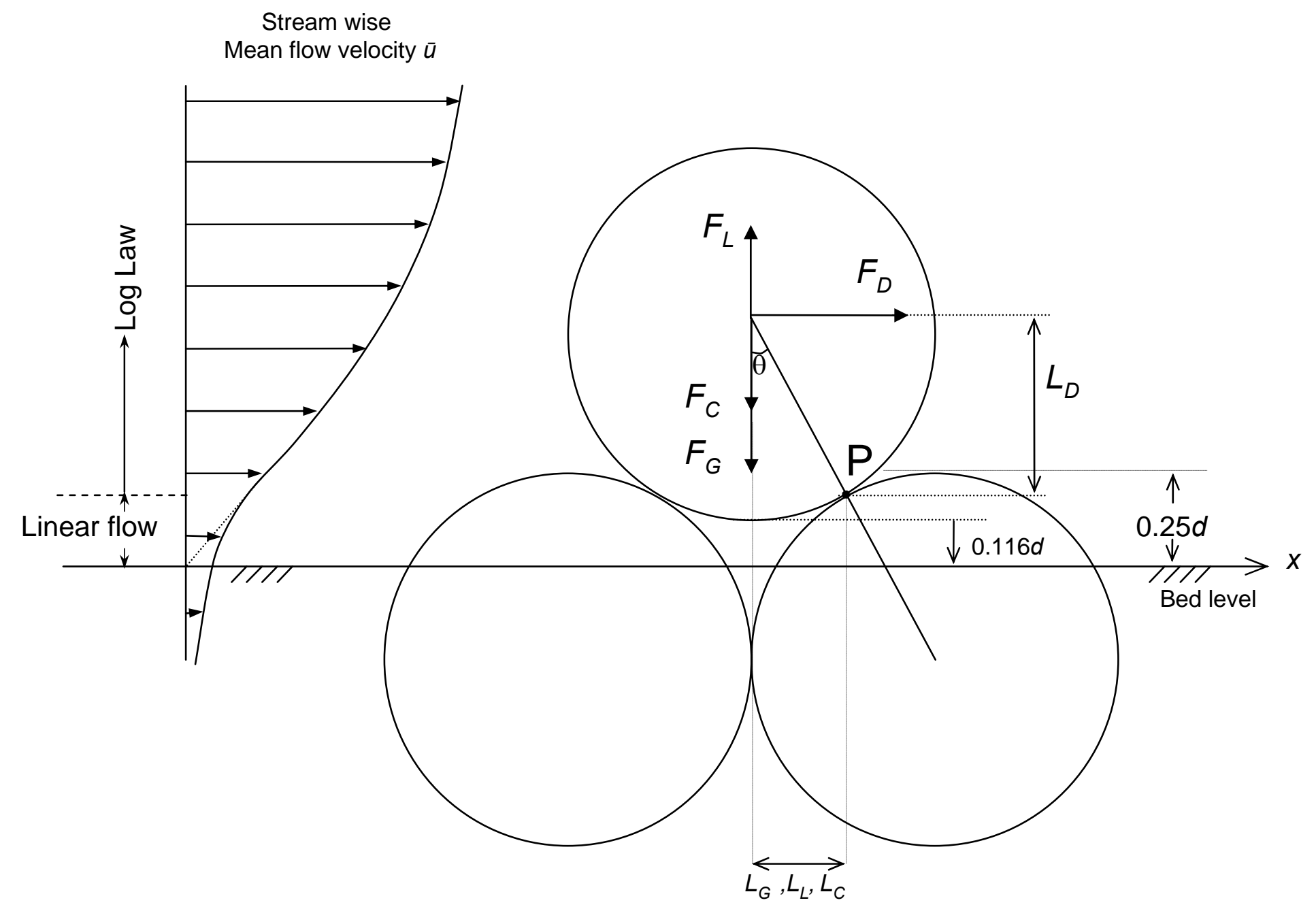

Figure 2 


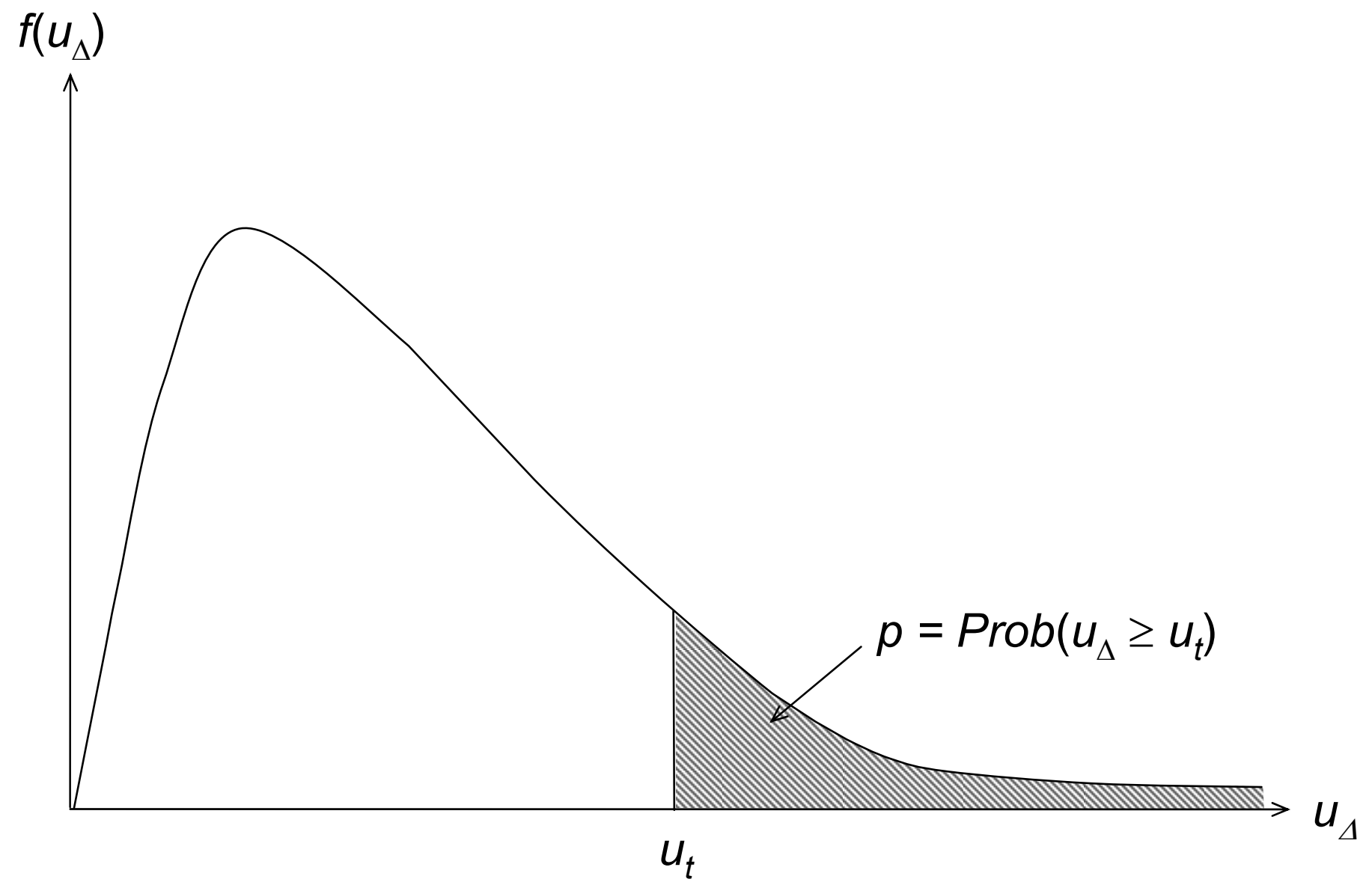

Figure 3 


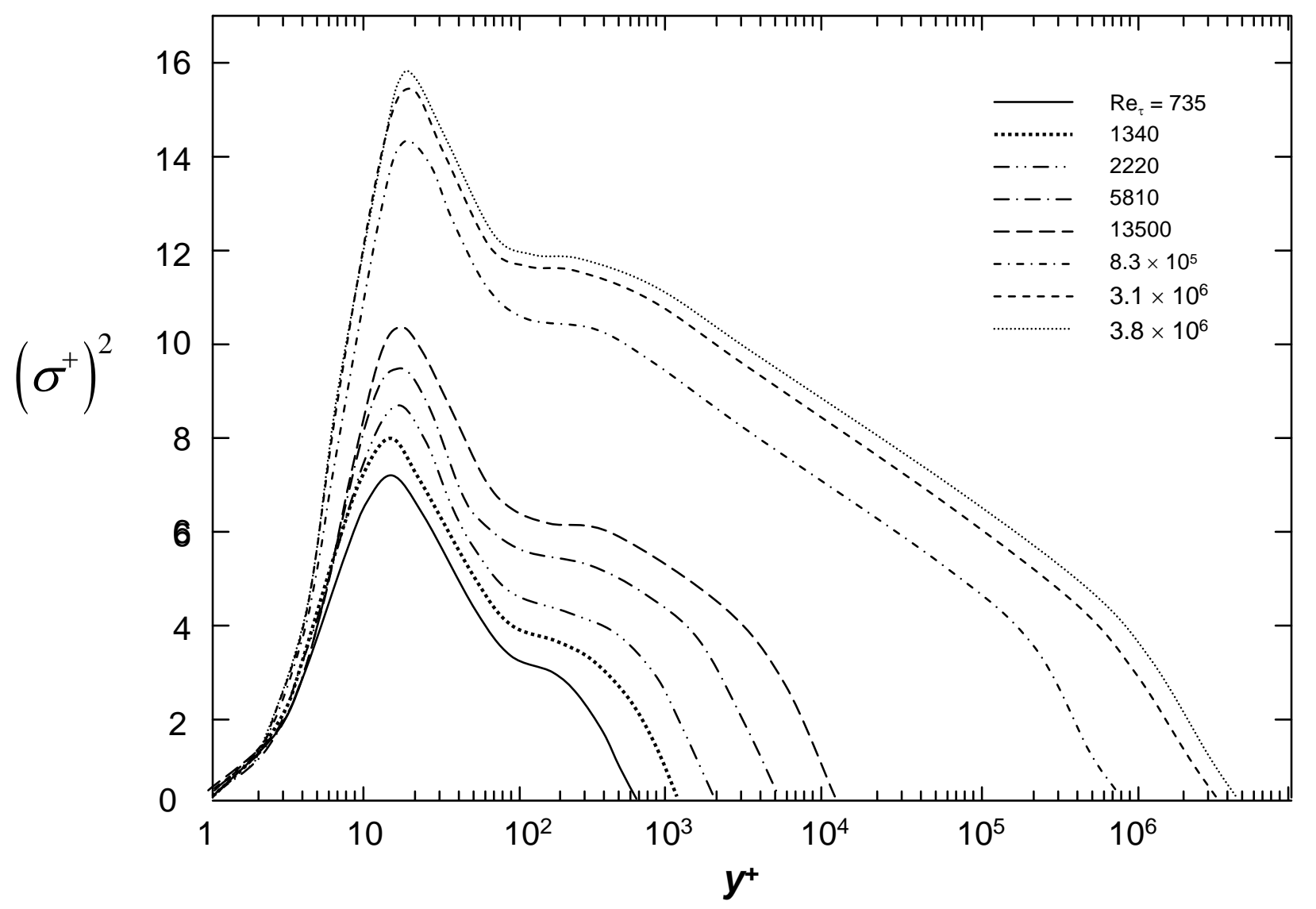

Figure 4 


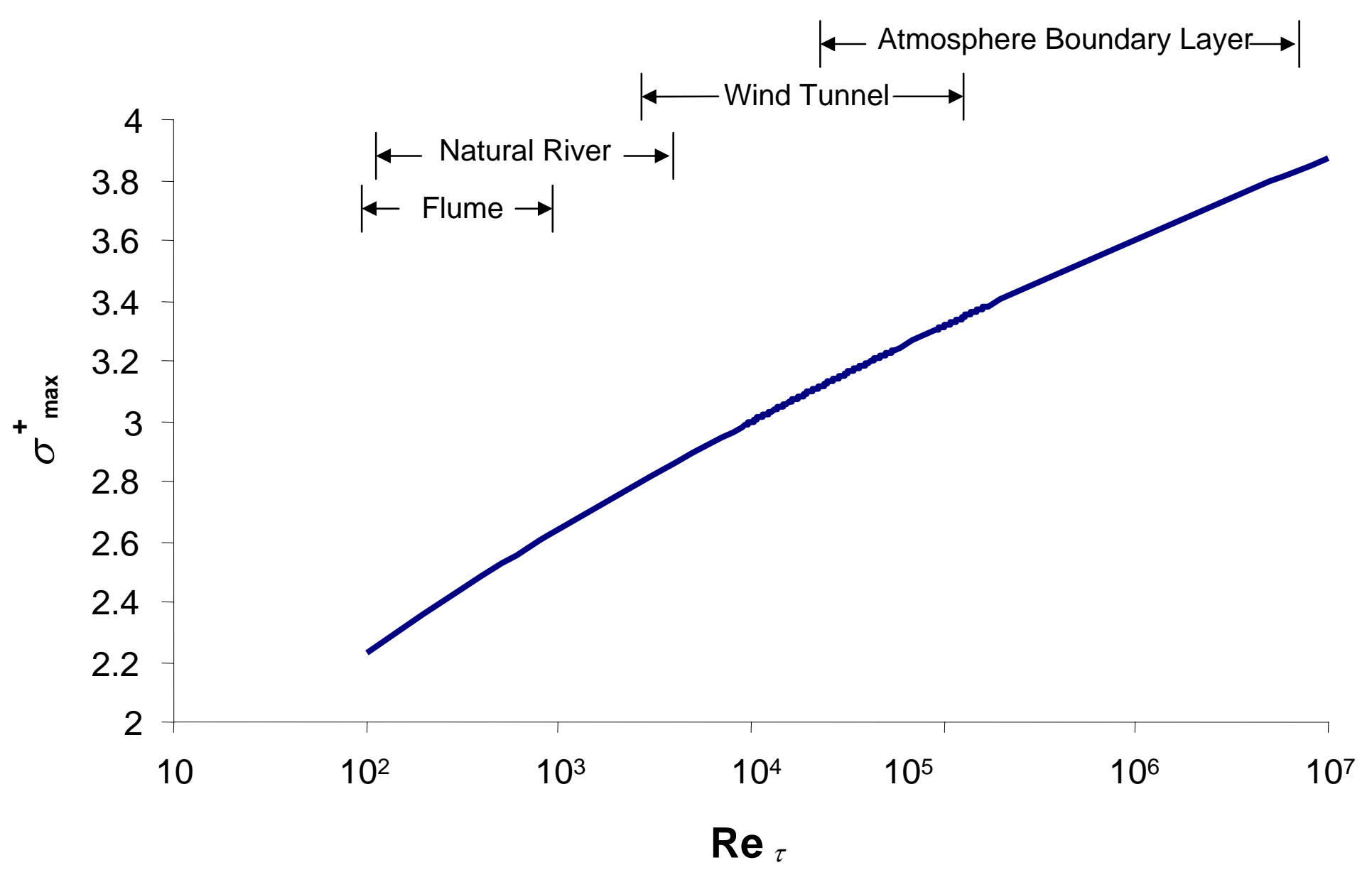

Figure 5 


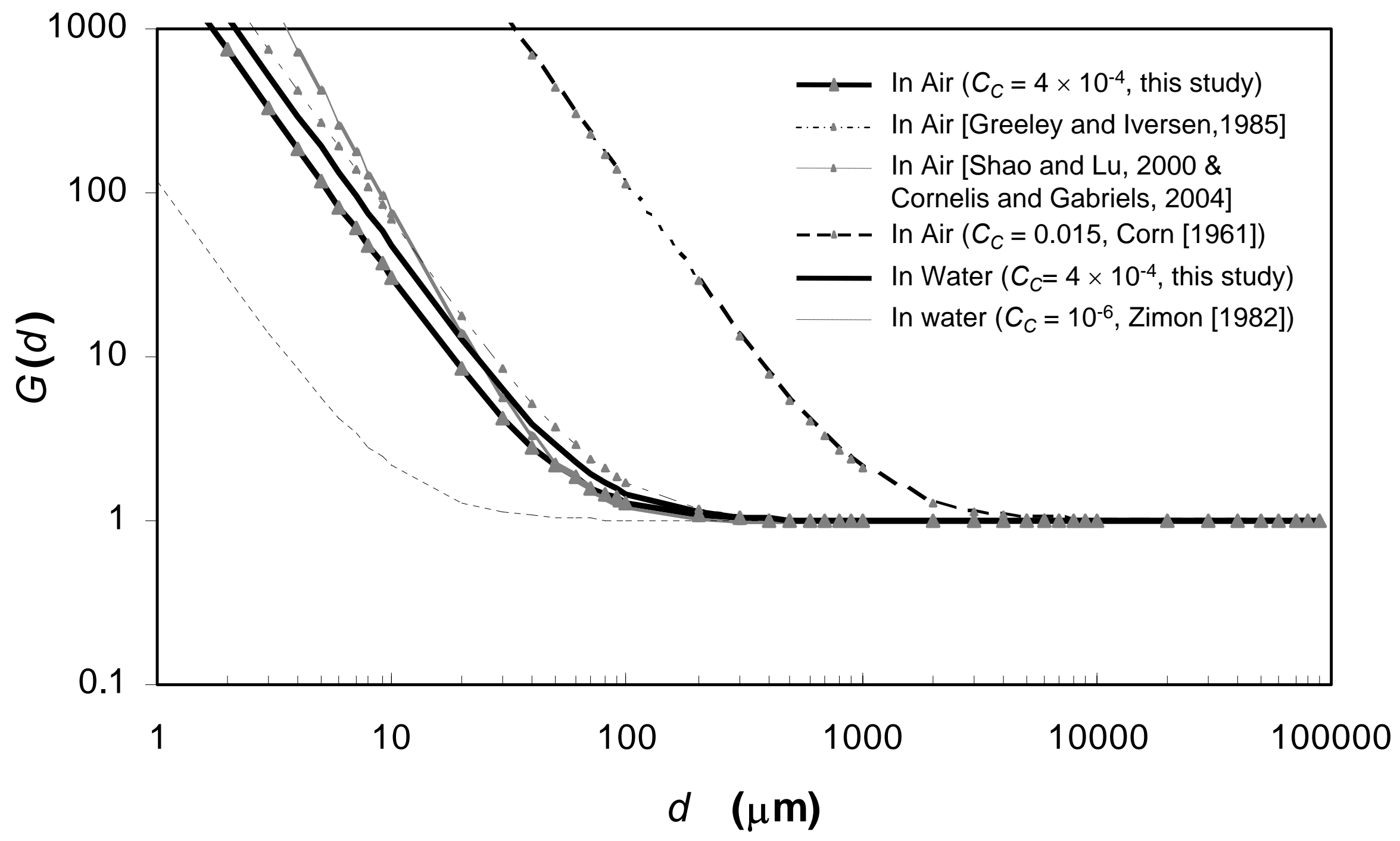

Figure 6 


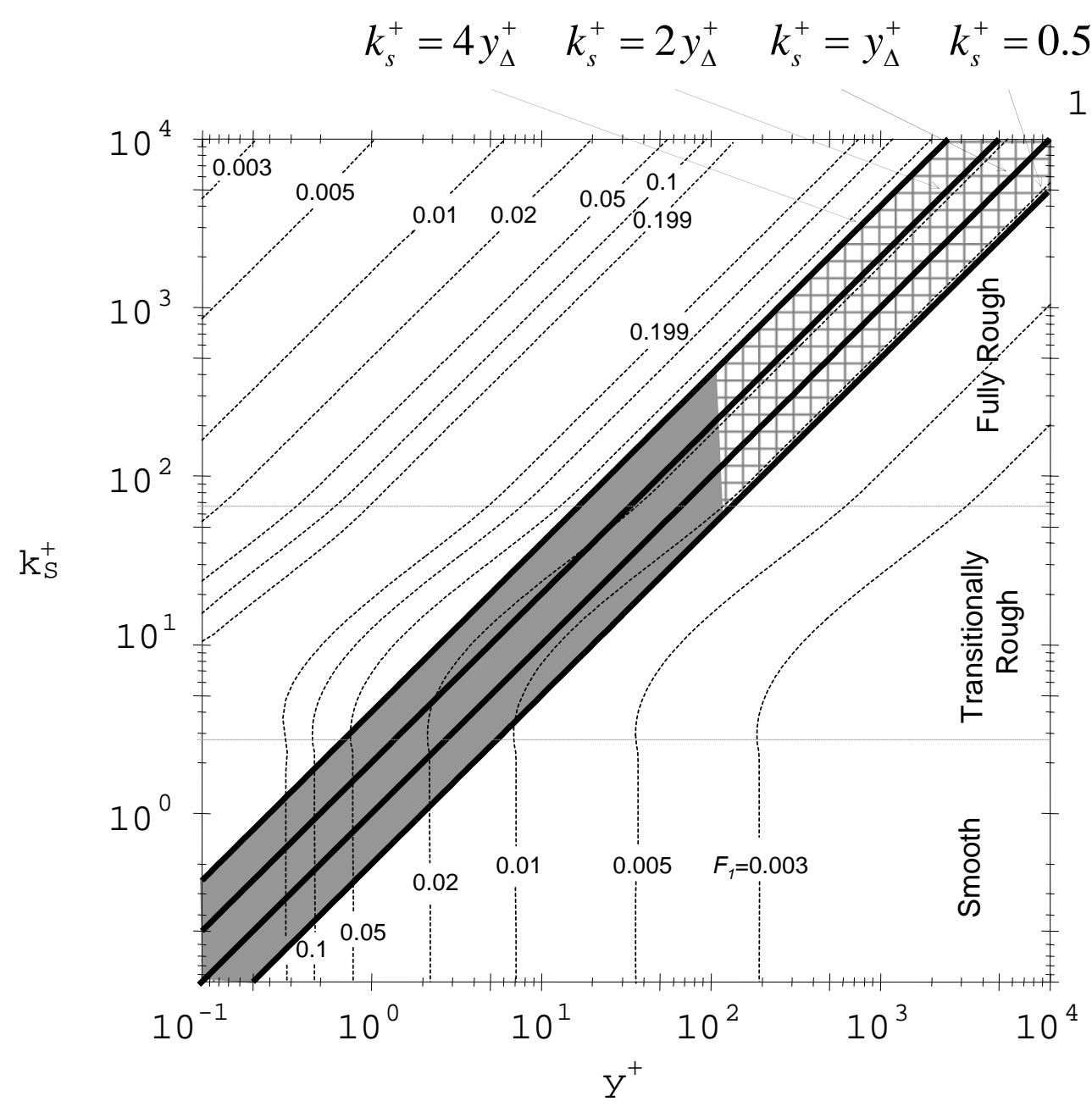

(a)

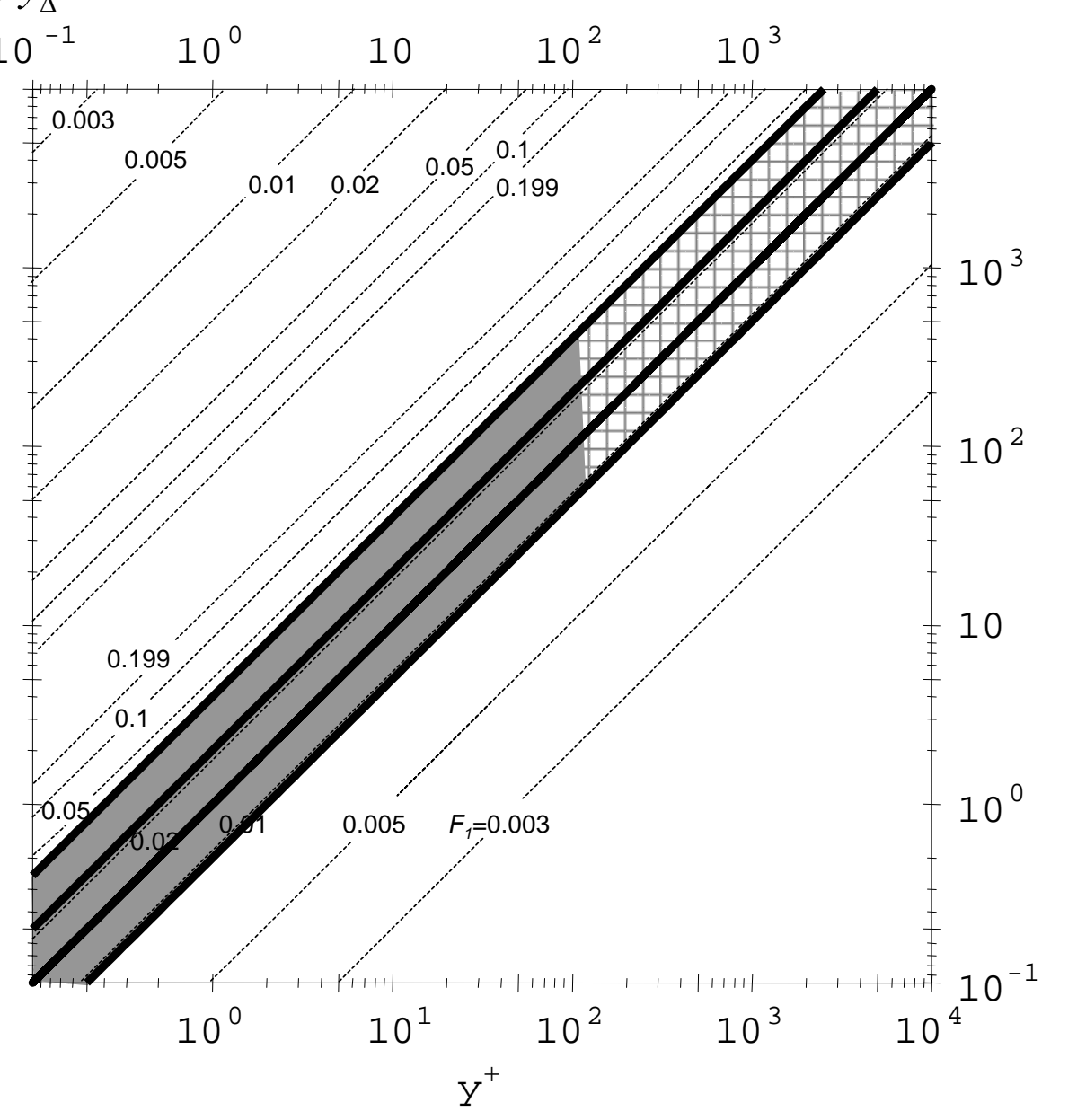

(b)

Figure 7 


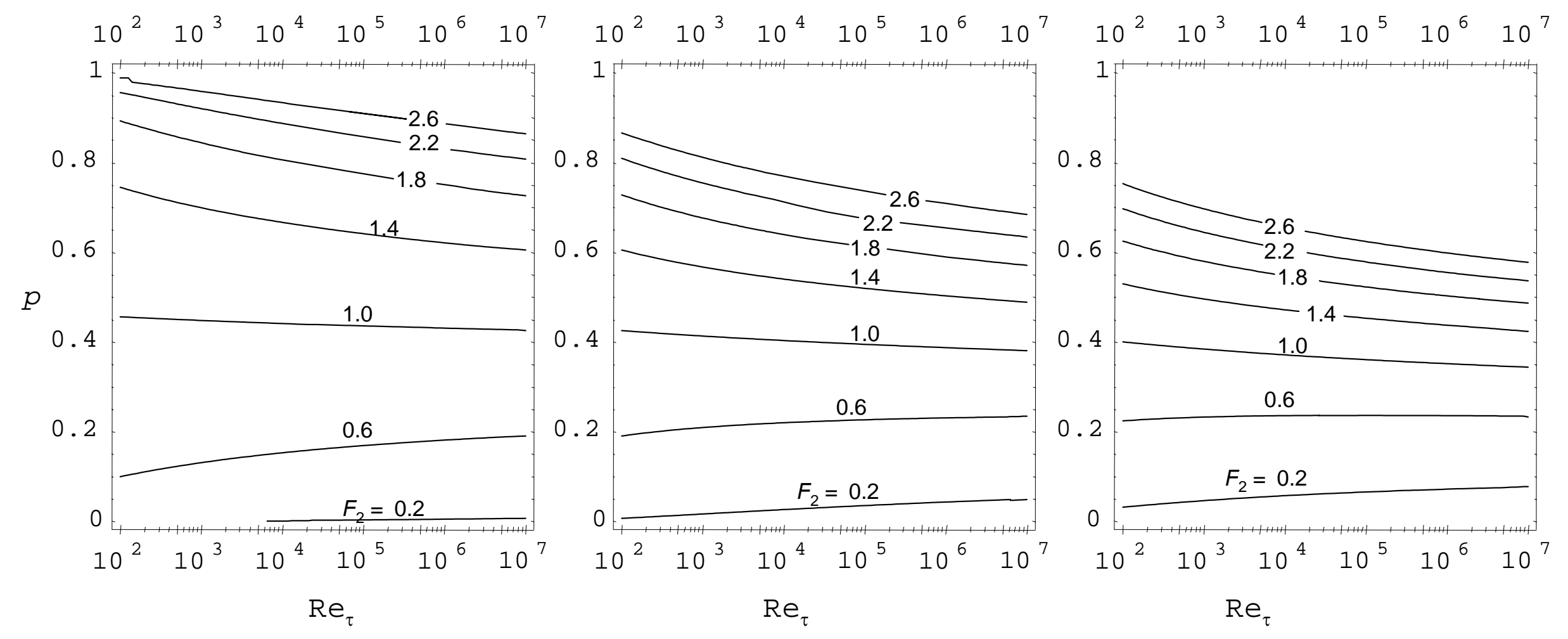

Figure 8 

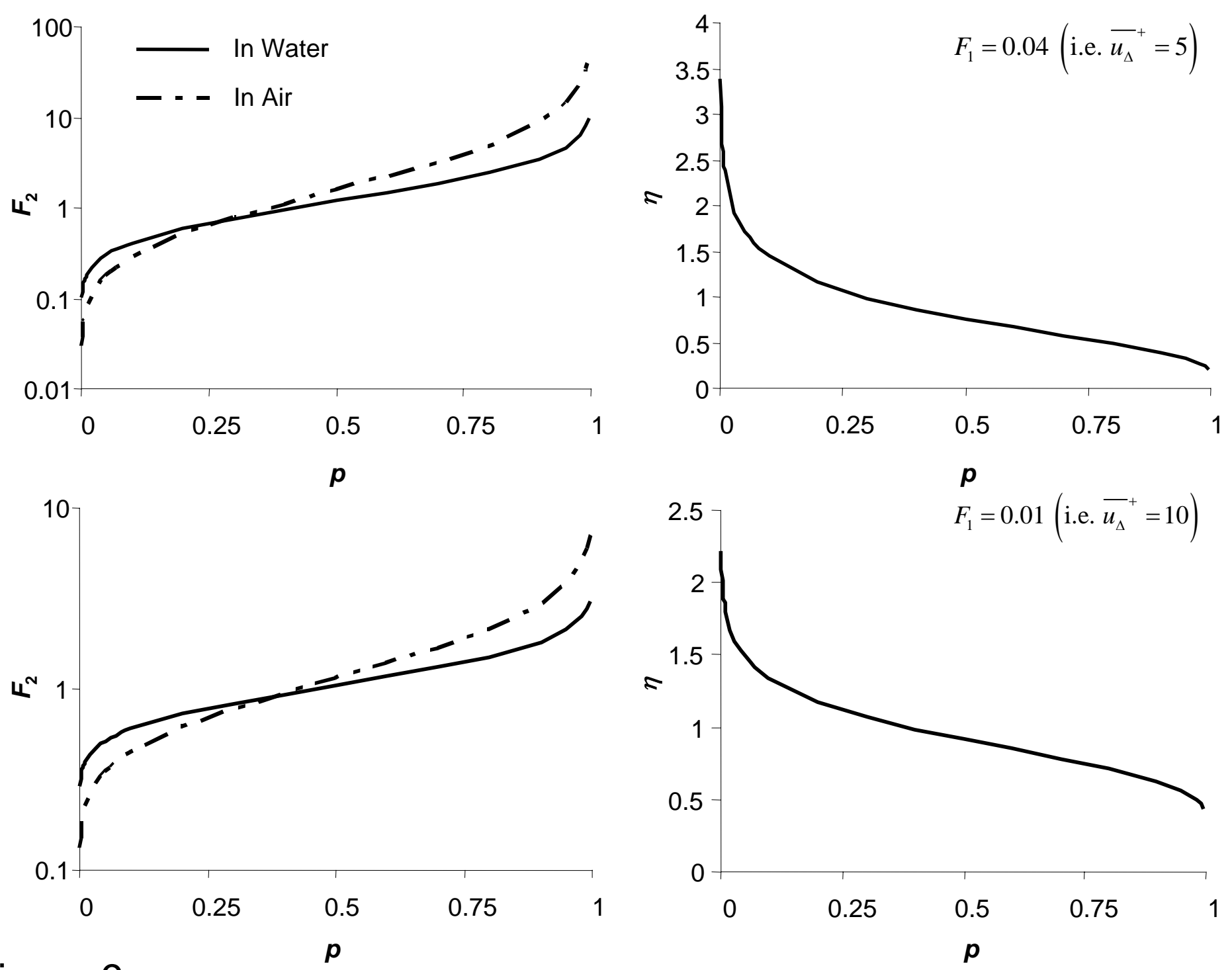

Figure 9 


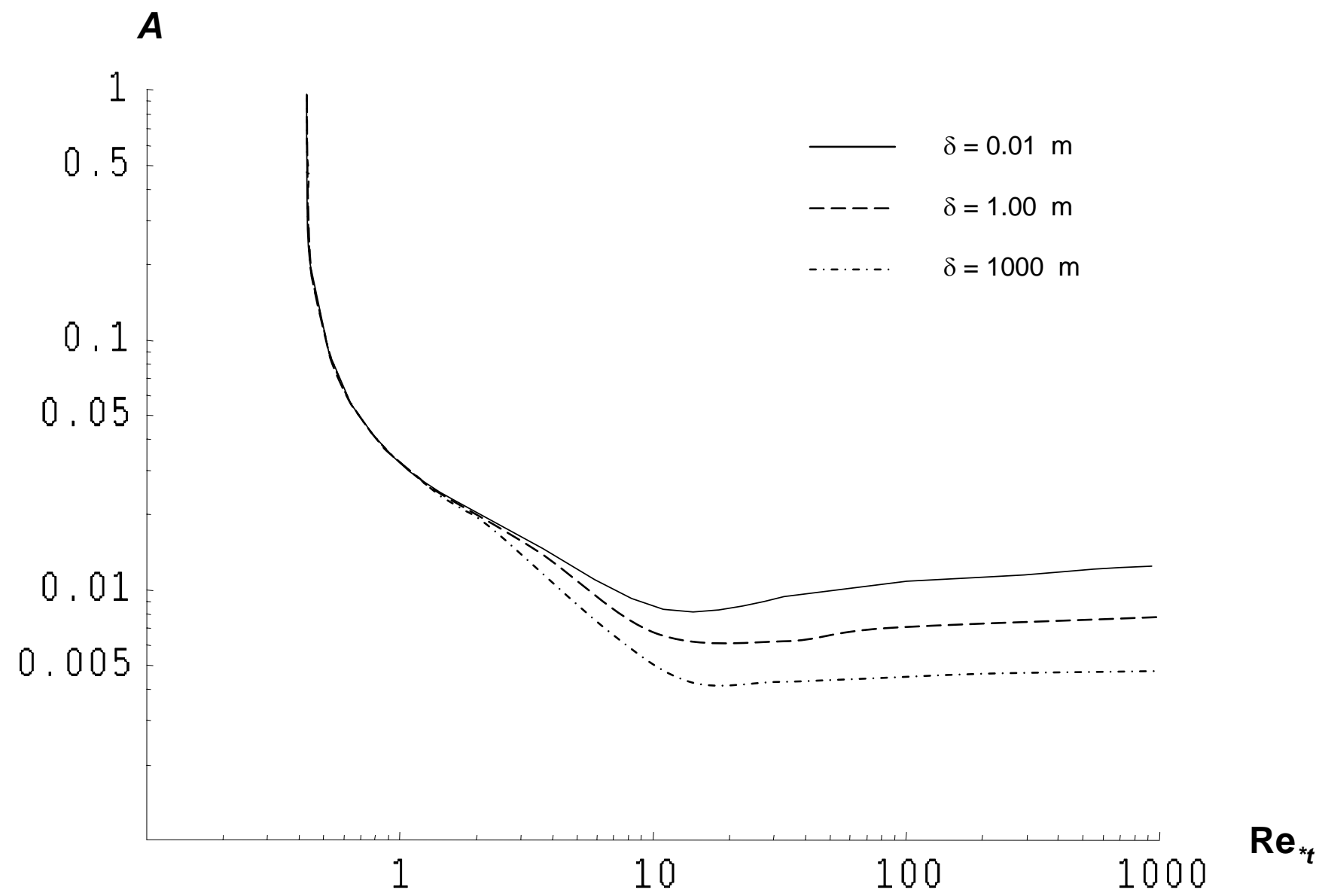

Figure 10 


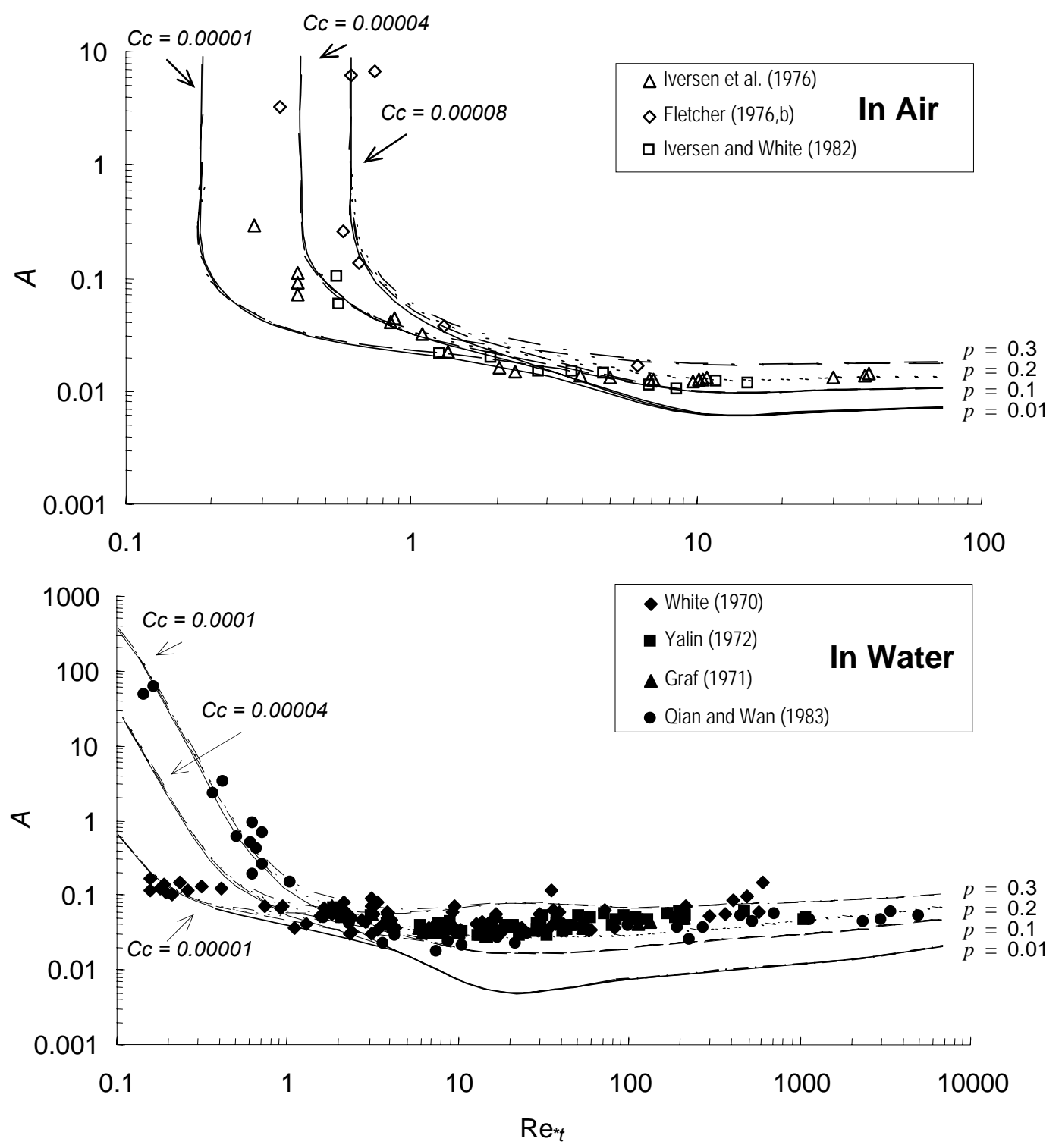

